\title{
The orientability problem in open Gromov-Witten theory
}

\author{
Penka Georgieva
}

\begin{abstract}
We give an explicit formula for the holonomy of the orientation bundle of a family of real Cauchy-Riemann operators. A special case of this formula resolves the orientability question for spaces of maps from Riemann surfaces with Lagrangian boundary condition. As a corollary, we show that the local system of orientations on the moduli space of $J$-holomorphic maps from a bordered Riemann surface to a symplectic manifold is isomorphic to the pullback of a local system defined on the product of the Lagrangian and its free loop space. As another corollary, we show that certain natural bundles over these moduli spaces have the same local systems of orientations as the moduli spaces themselves (this is a prerequisite for integrating the Euler classes of these bundles). We will apply these conclusions in future papers to construct and compute open Gromov-Witten invariants in a number of settings.
\end{abstract}

53D45, 57R17; 14N35

\section{Introduction}

The theory of $J$-holomorphic maps introduced by Gromov [5] plays a central role in the study of symplectic manifolds. Considerations in theoretical physics led to the development of the Gromov-Witten invariants. They are invariants of symplectic manifolds and can be interpreted as counts of $J$-holomorphic maps from a closed Riemann surface passing through prescribed constraints. Open string theory motivated the study of $J$-holomorphic maps from a bordered Riemann surface with boundary mapping to a Lagrangian submanifold and predicts the existence of open GromovWitten invariants. Their mathematical definition, however, has proved to be a subtle problem. Two main obstacles are the question of orientability and the existence of real codimension-one boundary strata of the moduli space of maps from a bordered Riemann surface. This work addresses the first of these issues.

The orientability question in the case $\Sigma=D^{2}$ was previously studied by de Silva [14] and Fukaya, Oh, Ohta and Ono [4]; see also Ekholm, Etnyre and Sullivan [2], Seidel [13], Wehrheim and Woodward [18] and Welschinger [19]. The moduli space of $J$-holomorphic maps from $D^{2}$ is not always orientable. However, Fukaya et al [4] showed that the moduli space is orientable if the Lagrangian $L$ is relatively spin and a 
choice of a relatively spin structure determines an orientation. This result was extended by Solomon [15] to relatively pin $^{ \pm}$Lagrangians and Riemann surfaces of higher genus with a fixed complex structure. Solomon constructed a canonical isomorphism between the determinant line bundle of the moduli space and the pullback by the evaluation maps of a certain number of copies of $\operatorname{det}(T L)$. We extend these results to any Lagrangian and allow the complex structure on the domain to vary. We also discover an entirely new topological condition sufficient for the orientability of the moduli space.

In this paper, we give an explicit criterion specifying whether the determinant line bundle of a loop of real Cauchy-Riemann operators over bordered Riemann surfaces is trivial; see Theorem 1.1. As a corollary, we conclude that the local system of orientations on the moduli space of $J$-holomorphic maps from a bordered Riemann surface is isomorphic to the pullback of a local system defined on the product of the Lagrangian and its free loop space; see Corollary 1.8. Our formula recovers the orientability results obtained in [4] and [15] as special cases and detects new topological conditions sufficient for the orientability of the moduli space, such as $w_{2}$ of an orientable Lagrangian being the square of a class; see Corollary 1.6. As another corollary, we show that the local systems of orientations of certain natural bundles over these moduli spaces are canonically isomorphic to the local systems of orientations of the moduli spaces themselves which is a prerequisite for integrating the Euler classes of these bundles; see Corollary 1.10. This is a generalization of Pandharipande, Solomon and Walcher [11, Lemma 13].

If $M$ is a manifold, possibly with boundary, or a (possibly nodal) surface, and $L \subset M$ is a submanifold, a bundle pair $(E, F) \rightarrow(M, L)$ consists of a complex vector bundle $E \rightarrow M$ and a maximal totally real subbundle $F \subset E_{\mid L}$. A real Cauchy-Riemann operator on a bundle pair $(E, F) \rightarrow(\Sigma, \partial \Sigma)$, where $\Sigma$ is an oriented surface with boundary $\partial \Sigma$, is a linear map of the form

$$
\begin{aligned}
D=\bar{\partial}+A: \Omega^{0}(E, F) \equiv\{\xi \in \Gamma(\Sigma, E) & : \xi(\partial \Sigma) \subset F\} \\
& \longrightarrow \Omega_{j}^{0,1}(E) \equiv \Gamma\left(\Sigma,\left(T^{*} \Sigma, j\right)^{0,1} \otimes_{\mathbb{C}} E\right),
\end{aligned}
$$

where $\bar{\partial}$ is the holomorphic $\bar{\partial}$-operator for some complex structure $j$ on $\Sigma$ compatible with the orientation and a holomorphic structure in $E$ and

$$
A \in \Gamma\left(\Sigma, \operatorname{Hom}_{\mathbb{R}}\left(E,\left(T^{*} \Sigma, j\right)^{0,1} \otimes_{\mathbb{C}} E\right)\right)
$$

is a zeroth-order deformation term. All real Cauchy-Riemann operators are Fredholm in appropriate completions; see McDuff and Salamon [10, Theorem C.1.10].

Let $I=[0,1]$. Given an orientation-preserving diffeomorphism $\phi: \Sigma \rightarrow \Sigma$, let

$$
\left(M_{\phi}, \partial M_{\phi}\right)=((\Sigma, \partial \Sigma) \times I) /(x, 1) \sim(\phi(x), 0)
$$


be the mapping torus of $\phi$ and $\pi: M_{\phi} \rightarrow S^{1}$ be the projection map. For each $t \in S^{1}$, let $\Sigma_{t}=\pi^{-1}(t)$ be the fiber over $t$. A continuous family of real Cauchy-Riemann operators on $(E, F)$ is a collection of real Cauchy-Riemann operators

$$
D_{t}: \Omega^{0}\left(E_{\mid \Sigma_{t}}, F_{\mid \partial \Sigma_{t}}\right) \rightarrow \Omega_{j_{t}}^{0,1}\left(E_{\mid \Sigma_{t}}\right)
$$

which varies continuously with $t \in S^{1}$. We denote by $\operatorname{det}(D) \rightarrow S^{1}$ the determinant line bundle corresponding to this family; see [10, Section A.2].

Theorem 1.1 Let $\Sigma$ be a smooth oriented bordered surface, $\phi: \Sigma \rightarrow \Sigma$ be a diffeomorphism preserving the orientation and each boundary component, and $(E, F)$ be a bundle pair over $\left(M_{\phi}, \partial M_{\phi}\right)$. For each boundary component $(\partial \Sigma)_{i}$ of $\Sigma$, choose a section $\alpha_{i}$ of

$$
\left(\partial M_{\phi}\right)_{i}=M_{\phi_{\mid(\partial \Sigma)_{i}}} \rightarrow S^{1} .
$$

If $D$ is any family of real Cauchy-Riemann operators on $(E, F)$, then

$$
\left\langle w_{1}(\operatorname{det} D), S^{1}\right\rangle=\sum_{i}\left(\left\langle w_{1}(F),(\partial \Sigma)_{i}\right\rangle+1\right)\left\langle w_{1}(F), \alpha_{i}\right\rangle+\sum_{i}\left\langle w_{2}(F),\left(\partial M_{\phi}\right)_{i}\right\rangle .
$$

Remark 1.2 Any two choices of sections $\alpha_{i}$ differ by a multiple of $(\partial \Sigma)_{i}$ and thus give the same $i^{\text {th }}$ term in the first sum above.

We prove this theorem in Section 3 by showing that the determinant line bundle of $D$ is isomorphic to the tensor product of the determinant line bundle of a $\bar{\partial}$-operator on a line bundle and the determinant line bundle of a $\bar{\partial}$-operator on an orientable bundle. The evaluation of their first Stiefel-Whitney classes then gives the two parts in the formula in Theorem 1.1.

The case of Theorem 1.1 when $\Sigma$ has one boundary component, $E \rightarrow \Sigma \times S^{1}$ is trivial, and $j$ is fixed is equivalent to the full statement of [13, Lemma 11.7], which expresses $w_{1}(\operatorname{det} D)$ in terms of characteristic classes of the Lagrangian Grassmannian $\operatorname{Gr}(n)=U(n) / O(n)$. Theorem 1.1 directly leads to orientability results for moduli spaces of bordered $J$-holomorphic maps; such results can be obtained from [13, Lemma 11.7] only in the case $\Sigma=D^{2}$ (and using the approach of Corollary 1.5). The way [13, Lemma 11.7] is generally used to study the orientation problem involves (relative) pin structures, covering only the known cases of [4] and [15]. As Theorem 1.1 and Corollary 1.5 show such a structure is not necessary for the orientability of the moduli spaces; for example, all moduli spaces associated to orientable Lagrangians with $w_{2}$ being a square class are orientable, regardless of whether or not they are relatively spin. The last result is clearly visible from Theorem 1.1, but not from [13, Lemma 11.7] even in the disk case. The proof of [13, Lemma 11.7] involves explicitly 
determining generators for $\pi_{1}$ of the free loop space of $\operatorname{Gr}(n)$, which then give rise to the two terms of the righthand side in [13, (11.16)]. Our proof of Theorem 1.1 provides a completely different and more geometric decomposition of det $D$ and applies to the general setting, from both the topological viewpoint ( $E \rightarrow M_{\phi}$ need not be trivial) and the analytic one ( $j$ need not be fixed).

Remark 1.3 Families of real Cauchy-Riemann operators often arise by pulling back data from a target manifold by smooth maps as follows. Suppose $(M, J)$ is an almost complex manifold, $L \subset M$ is a submanifold, $(E, \mathfrak{I}) \rightarrow M$ is a complex vector bundle, and $F \subset E_{\mid L}$ is a maximal totally real subbundle. Let $\nabla$ be a connection in $(E, \mathfrak{I})$ and

$$
A \in \Gamma\left(M, \operatorname{Hom}_{\mathbb{R}}\left(E, T^{*} M^{0,1} \otimes_{\mathbb{C}} E\right)\right) .
$$

For any bordered Riemann surface $(\Sigma, j)$ and any map $u:(\Sigma, \partial \Sigma) \rightarrow(M, L)$, let $\nabla^{u}$ denote the induced connection in $u^{*} E$ and

$$
A_{u}=A \circ \partial u \in \Gamma\left(\Sigma, \operatorname{Hom}\left(u^{*} E,\left(T^{*} \Sigma, j\right)^{0,1} \otimes_{\mathbb{C}} u^{*} E\right)\right) .
$$

The homomorphisms

$$
\bar{\partial}_{u}^{\nabla}=\frac{1}{2}\left(\nabla^{u}+\mathfrak{I} \circ \nabla^{u} \circ j\right), \quad \bar{\partial}_{u}^{\nabla}+A_{u}: \Omega^{0}\left(u^{*} E, u_{\mid \partial \Sigma}^{*} F\right) \rightarrow \Omega_{j}^{0,1}\left(u^{*} E\right)
$$

are then real Cauchy-Riemann operators that form families of real Cauchy-Riemann operators over families of maps.

Throughout this paper, we denote by $(M, \omega)$ a symplectic manifold, by $L \subset M$ a Lagrangian submanifold, and by $J$ a tame almost complex structure on $M$. Fix a tuple of homology classes

$$
\boldsymbol{b}=\left(b, b_{1}, \ldots, b_{h}\right) \in H_{2}(M, L) \oplus H_{1}(L)^{\oplus h},
$$

an oriented bordered surface $(\Sigma, \partial \Sigma)$ of genus $g$, an ordering of the boundary components

$$
\partial \Sigma=\coprod_{i=1}^{h}(\partial \Sigma)_{i} \cong \coprod_{i=1}^{h} S^{1},
$$

a nonnegative integer $l$, and a tuple $\boldsymbol{k}=\left(k_{1}, \ldots, k_{h}\right) \in \mathbb{Z}_{+}^{h}$. Let $\mathfrak{B}_{l, \boldsymbol{k}}^{g, h}(M, L, \boldsymbol{b})$ be the space of tuples $\left(u, z, x_{1}, \ldots, x_{h}\right)$, where

- $z$ is a tuple of $l$ interior marked points,

- $\boldsymbol{x}_{i}$ is a tuple of $k_{i}$ marked points on $(\partial \Sigma)_{i}$,

- $u$ is a map from $(\Sigma, \partial \Sigma)$ to $(M, L)$, which represents the class $b \in H_{2}(M, L)$ and for which the restriction $u_{\mid(\partial \Sigma)_{i}}$ represents the class $b_{i} \in H_{1}(L)$. 
In this definition, we can consider either smooth maps $u$ or maps of class $W^{1, p}$, with $p>2$. Let $\mathcal{J}_{\Sigma}$ be the space of complex structures on $\Sigma$ compatible with the orientation, $\mathcal{D}$ be the diffeomorphism group of $\Sigma$ preserving the orientation and each boundary component, and

$$
\mathcal{H}_{l, \boldsymbol{k}}^{g, h}(M, L, \boldsymbol{b})=\left(\mathfrak{B}_{l, \boldsymbol{k}}^{g, h}(M, L, \boldsymbol{b}) \times \mathcal{J}_{\Sigma}\right) / \mathcal{D}
$$

Remark 1.4 Throughout the paper we assume that the action of $\mathcal{D}$ has no fixed points. Thus, the quotient space $\mathcal{H}_{l, \boldsymbol{k}}^{g, h}(M, L, \boldsymbol{b})$ is a topological manifold. This happens for example if there are sufficiently many marked points. In applications to more general cases, this issue can be avoided by working with Prym structures on Riemann surfaces; see Looijenga [8].

The determinant line bundle of a family of real Cauchy-Riemann operators $D_{(E, F)}$ on $\mathfrak{B}_{l, \boldsymbol{k}}^{g, h}(M, L, \boldsymbol{b}) \times \mathcal{J}_{\Sigma}$ induced by a bundle pair $(E, F)$ as in Remark 1.3 descends to a line bundle over $\mathcal{H}_{l, \boldsymbol{k}}^{g, h}(M, L, \boldsymbol{b})$, which we denote by det $D_{(E, F)}$. As a direct corollary of Theorem 1.1 , we obtain the following result on its orientability.

Corollary 1.5 Let $\gamma$ be a loop in $\mathcal{H}_{l, \boldsymbol{k}}^{g, h}(M, L, \boldsymbol{b})$ and $\widetilde{\gamma}$ a path in $\mathfrak{B}_{l, \boldsymbol{k}}^{g, h}(M, L, \boldsymbol{b}) \times \mathcal{J}_{\Sigma}$ lifting $\gamma$ such that $\widetilde{\gamma}_{1}=\phi \cdot \widetilde{\gamma}_{0}$ for some $\phi \in \mathcal{D}$ with $\phi_{\mid \partial \Sigma}=\mathrm{id}$. For each boundary component $(\partial \Sigma)_{i}$ of $\Sigma$, denote by $\alpha_{i}: S^{1} \rightarrow L$ and $\beta_{i}: S^{1} \times(\partial \Sigma)_{i} \rightarrow L$ the paths traced by a fixed point on $(\partial \Sigma)_{i}$ and by the entire boundary component $(\partial \Sigma)_{i}$. Then,

$(1-2)\left\langle w_{1}\left(\operatorname{det} D_{(E, F)}\right), \gamma\right\rangle=\sum_{i=1}^{h}\left(\left\langle w_{1}(F), b_{i}\right\rangle+1\right)\left\langle w_{1}(F), \alpha_{i}\right\rangle+\sum_{i=1}^{h}\left\langle w_{2}(F), \beta_{i}\right\rangle$.

By Lemma 2.4, every loop $\gamma$ in $\mathcal{H}_{l, \boldsymbol{k}}^{g, h}(M, L, \boldsymbol{b})$ admits a lift $\widetilde{\gamma}$ such that $\widetilde{\gamma}_{1}=\phi \cdot \widetilde{\gamma}_{0}$ for some $\phi \in \mathcal{D}$ with $\phi_{\mid \partial \Sigma}=\mathrm{id}$. Thus, the first assumption in Corollary 1.5 imposes no restriction on $\gamma$.

Corollary 1.6 Let $\gamma$ and $\alpha_{i}$ be as in Corollary 1.5. If for some $\sigma \in H^{1}(L)$ the class $w_{2}(F)+\sigma^{2}$ belongs to $\operatorname{Im}\left(i^{*}: H^{2}(M) \rightarrow H^{2}(L)\right)$, then

$$
\left\langle w_{1}\left(\operatorname{det}\left(D_{(E, F)}\right)\right), \gamma\right\rangle=\sum_{i=1}^{h}\left(\left\langle w_{1}(F), b_{i}\right\rangle+1\right)\left\langle w_{1}(F), \alpha_{i}\right\rangle .
$$

In particular, if $F$ is also orientable or $\left\langle w_{1}(F), b_{i}\right\rangle=1$ for every $i=1, \ldots, h$, then $\operatorname{det}\left(D_{(E, F)}\right)$ is orientable. 
The presence of $w_{2}(F)$ in (1-2) means that in general the local system of orientations on $\operatorname{det}\left(D_{(E, F)}\right)$ is not the pullback of a system on $L$. In Section 4 , we construct a local system $\mathcal{Z}_{\left(w_{1}, w_{2}\right)}^{F}$ on the $h$-fold product of the Lagrangian and its free loop space $\mathcal{L}(L)$, which traces the twisting coming from the righthand side of (1-2). When there is at least one boundary marked point on each boundary component, there is a natural map

$$
\text { ev: } \mathcal{H}_{l, \boldsymbol{k}}^{g, h}(M, L, \boldsymbol{b}) \rightarrow(L \times \mathcal{L}(L))^{h},
$$

which is canonically determined up to homotopy; see Proposition 4.6. We show that the pullback of $\mathcal{Z}_{\left(w_{1}, w_{2}\right)}^{F}$ under this map is isomorphic to the local system twisted by the first Stiefel-Whitney class of $\operatorname{det}\left(D_{(E, F)}\right)$. Moreover, ev* $\mathcal{Z}_{\left(w_{1}, w_{2}\right)}^{F}$ is trivial along the fiber of the map forgetting the boundary marked point(s) and pushes down to the space with no boundary marked points; see Lemma 4.7. Depending on the context, denote by $\widetilde{\mathrm{ev}}^{*} \mathcal{Z}_{\left(w_{1}, w_{2}\right)}^{F}$ either the pulled-back system or its pushdown under the forgetful map.

Theorem 1.7 There is a local system $\mathcal{Z}_{\left(w_{1}, w_{2}\right)}^{F}$ on $(L \times \mathcal{L}(L))^{h}$ such that the local system of orientations of $\operatorname{det} D_{(E, F)}$ is isomorphic to $\widetilde{\mathrm{ev}}^{*} \mathcal{Z}_{\left(w_{1}, w_{2}\right)}^{F}$. An isomorphism between the two systems is determined by a choice of a trivialization of $F$ over a basepoint in $L$ and trivializations of $F \oplus 3 \operatorname{det}(F)$ over representatives for the homotopy classes of loops in $L$.

The $\bar{\partial}$-operator on $\mathfrak{B}_{l, \boldsymbol{k}}^{g, h}(M, L, \boldsymbol{b}) \times \mathcal{J}_{\Sigma}$, given by

$$
\bar{\partial}(u, j)=\frac{1}{2}(d u+J \circ d u \circ j),
$$

descends to $\mathcal{H}_{l, \boldsymbol{k}}^{g, h}(M, L, \boldsymbol{b})$. The moduli space $\mathfrak{M}_{l, \boldsymbol{k}}^{g, h}(M, L, \boldsymbol{b}) \subset \mathcal{H}_{l, \boldsymbol{k}}^{g, h}(M, L, \boldsymbol{b})$ consists of elements $\left[u, z, x_{1}, \ldots, x_{h}\right]$ satisfying $\bar{\partial} u=0$. Linearizations of the $\bar{\partial}-$ operator along $\mathfrak{B}_{l, \boldsymbol{k}}^{g, h}(M, L, \boldsymbol{b})$ are real Cauchy-Riemann operators over $\Sigma$ induced by the bundle pair $(T M, T L) \rightarrow(M, L)$; see [10, Section 3.1]. Their determinant line bundle descends to a line bundle over $\mathcal{H}_{l, \boldsymbol{k}}^{g, h}(M, L, \boldsymbol{b})$, which we $\operatorname{denote}$ by $\operatorname{det}\left(D_{\bar{\partial}}\right)$. The significance of this bundle comes from the fact that when the moduli space is cut transversely, the top exterior power of its tangent bundle is essentially the bundle $\operatorname{det}\left(D_{\bar{\partial}}\right)$. As a corollary of Theorem 1.7 , we obtain the following statement concerning the orientation system of this moduli space.

Corollary 1.8 There is a local system $\mathcal{Z}_{\left(w_{1}, w_{2}\right)}^{T L}$ on $(L \times \mathcal{L}(L))^{h}$ such that the local system of orientations on $\mathfrak{M}_{l, \boldsymbol{k}}^{g, h}(M, L, \boldsymbol{b})$ is isomorphic to $\widetilde{\mathrm{ev}}^{*} \mathcal{Z}_{\left(w_{1}, w_{2}\right)}^{T L}$. An isomorphism between the two systems is determined by a choice of a trivialization of $T L$ over a basepoint in $L$ and trivializations of $T L \oplus 3 \operatorname{det}(T L)$ over representatives for the homotopy classes of loops in $L$. 
Remark 1.9 By Corollaries 1.6 and 1.8, $\mathfrak{M}_{l, \boldsymbol{k}}^{g, h}(M, L, \boldsymbol{b})$ is orientable if $w_{2}(L)+\sigma^{2}$ is in the image of the restriction homomorphism $H^{2}(M) \rightarrow H^{2}(L)$ for some $\sigma \in H^{1}(L)$ and $L$ is orientable; with $\sigma=0$, this recovers [4, Theorem 8.1.1]. If $L \subset M$ is not orientable, but $w_{2}(L)$ is as before, the orientation system of $\mathfrak{M}_{l, \boldsymbol{k}}^{g, h}(M, L, \boldsymbol{b})$ is a pullback/pushdown of several copies of the orientation system of the Lagrangian $L$; with $\sigma=0, w_{1}(L)$, this recovers [15, Theorem 1.1].

An important collection of examples of operators $D_{(E, F)}$ arises as follows. If $\boldsymbol{a}=\left(a_{1}, \ldots, a_{m}\right)$ is an $m$-tuple of positive integers and $n \in \mathbb{Z}^{+}$, let

$$
\mathcal{L}_{n, \boldsymbol{a}}=\mathcal{O}_{\mathbb{C} \mathbb{P}^{n}}\left(a_{1}\right) \oplus \cdots \oplus \mathcal{O}_{\mathbb{C P}^{n}}\left(a_{m}\right) \rightarrow \mathbb{C P}^{n}
$$

The natural conjugation on $\mathbb{C} \mathbb{P}^{n}$ lifts to $\mathcal{L}_{n, \boldsymbol{a}}$. Denote its fixed locus by $\mathcal{L}_{n, \boldsymbol{a}}^{\mathbb{R}}$; this is a real vector bundle over $\mathbb{R} \mathbb{P}^{n}$. Let

$$
\pi_{n, \boldsymbol{a}}: \mathcal{V}_{n, \boldsymbol{a}}^{\mathbb{R}}=\mathfrak{M}_{l, \boldsymbol{k}}^{g, h}\left(\mathcal{L}_{n, \boldsymbol{a}}, \mathcal{L}_{n, \boldsymbol{a}}^{\mathbb{R}}, \boldsymbol{b}\right) \rightarrow \mathfrak{M}_{l, \boldsymbol{k}}^{g, h}\left(\mathbb{C} \mathbb{P}^{n}, \mathbb{R} \mathbb{P}^{n}, \boldsymbol{b}\right) .
$$

The fiber of $\pi_{n, \boldsymbol{a}}$ over $\left[u, \boldsymbol{z}, \boldsymbol{x}_{\mathbf{1}}, \ldots, \boldsymbol{x}_{\boldsymbol{h}}\right]$ is canonically isomorphic to

$$
\operatorname{Ker} \bar{\partial}_{\left(u^{*} \mathcal{L}_{n, \boldsymbol{a}}, u^{*} \mathcal{L}_{n, \boldsymbol{a}}^{\mathbb{R}}\right)} \text {. }
$$

By [10, Theorem C.1.10(iii)], $\bar{\partial}_{\left(u^{*} \mathcal{L}_{n, \boldsymbol{a}}, u^{*} \mathcal{L}_{n, \boldsymbol{a}}^{\mathbb{R}}\right)}$ is surjective if $\mu(b) \geq 4 g+2 h-2$. Thus, $\mathcal{V}_{n, \boldsymbol{a}}^{\mathbb{R}}$ is a vector bundle in this case and its orientation line bundle agrees with $\operatorname{det}\left(\bar{\partial}_{\left(u^{*} \mathcal{L}_{n, \boldsymbol{a}}, u^{*} \mathcal{L}_{n, \boldsymbol{a}}^{\mathbb{R}}\right)}\right)$. The following corollary of Theorem 1.7 , suggests that it may be possible to integrate the twisted Euler class $e\left(\mathcal{V}_{n, \boldsymbol{a}}^{\mathbb{R}}\right)$ against the homology class of a compactification

$$
\widetilde{\mathfrak{M}}_{l, \boldsymbol{k}}^{g, h}\left(\mathbb{C} \mathbb{P}^{n}, \mathbb{R}^{n}, \boldsymbol{b}\right) \quad \text { of } \quad \mathfrak{M}_{l, \boldsymbol{k}}^{g, h}\left(\mathbb{C} \mathbb{P}^{n}, \mathbb{R}^{n}, \boldsymbol{b}\right)
$$

in some cases, including when the corresponding complete intersection $X_{n, \boldsymbol{a}}$ is a Calabi-Yau threefold; see Remark 1.11.

Corollary 1.10 Let $m, n \in \mathbb{Z}^{+}$and $\boldsymbol{a} \in\left(\mathbb{Z}^{+}\right)^{m}$ be such that $n-\sum a_{i}$ is odd. Let $\boldsymbol{b}$ be as in (1-1) with $(M, L)=\left(\mathbb{C} \mathbb{P}^{n}, \mathbb{R P}^{n}\right)$. If $\mu(b) \geq 4 g+2 h-2$, the line bundles

$$
\bigwedge_{\mathbb{R}}^{\text {top }} \mathcal{V}_{n ; \boldsymbol{a}}^{\mathbb{R}}, \quad \bigwedge_{\mathbb{R}}^{\text {top }} T \mathfrak{M}_{l, \boldsymbol{k}}^{g, h}\left(\mathbb{C} \mathbb{P}^{n}, \mathbb{R P}^{n}, \boldsymbol{b}\right) \rightarrow \mathfrak{M}_{l, \boldsymbol{k}}^{g, h}\left(\mathbb{C} \mathbb{P}^{n}, \mathbb{R} \mathbb{P}^{n}, \boldsymbol{b}\right)
$$

are canonically isomorphic up to multiplication by $\mathbb{R}^{+}$in each fiber.

Remark 1.11 If $s \in H^{0}\left(\mathbb{C} \mathbb{P}^{n}, \mathcal{L}_{n, a}\right)$ is a transverse section commuting with the conjugations on $\mathbb{C} \mathbb{P}^{n}$ and $\mathcal{L}_{n, \boldsymbol{a}}, X_{n, \boldsymbol{a}}=s^{-1}(0)$ is a smooth complete intersection with conjugation inherited from $\mathbb{C} \mathbb{P}^{n}$. The section $s$ induces a section $\widetilde{s}$ of (1-4) such that

$$
\mathfrak{M}_{l, \boldsymbol{k}}^{g, h}\left(X_{n, \boldsymbol{a}}, X_{n, \boldsymbol{a}}^{\mathbb{R}}, \boldsymbol{b}\right)=\tilde{s}^{-1}(0) \subset \mathfrak{M}_{l, \boldsymbol{k}}^{g, h}\left(\mathbb{C} \mathbb{P}^{n}, \mathbb{R} \mathbb{P}^{n}, \boldsymbol{b}\right),
$$


where

$$
X_{n, \boldsymbol{a}}^{\mathbb{R}}=X_{n, \boldsymbol{a}} \cap \mathbb{R}^{n}
$$

This suggests that (open) Gromov-Witten invariants of $\left(X_{n, \boldsymbol{a}}, X_{n, \boldsymbol{a}}^{\mathbb{R}}\right)$, which should arise from the moduli space $\mathfrak{M}_{l, \boldsymbol{k}}^{g, h}\left(X_{n, \boldsymbol{a}}, X_{n, \boldsymbol{a}}^{\mathbb{R}}, \boldsymbol{b}\right)$, can be computed by integrating the Euler class

$$
e\left(\mathcal{V}_{n, \boldsymbol{a}}^{\mathbb{R}}\right) \text { against }\left[\widetilde{\mathfrak{M}}_{l, \boldsymbol{k}}^{g, h}\left(\mathbb{C} \mathbb{P}^{n}, \mathbb{R}^{n}, \boldsymbol{b}\right)\right]
$$

which can be done via equivariant localization. By Bini, de Concini, Polito and Procesi [1, Section 2.1.2]; Li and Zinger [7, Theorem 1.1]; and [11, Theorem 3], this is indeed the case if $g+h \leq 1$ and $X_{n, \boldsymbol{a}}$ is a Calabi-Yau threefold in the $h=1$ case. Based on Walcher [17] and Popa and Zinger [12], there are strong indications that this is also the case for $(g, h)=(0,2)$. We plan to investigate this in the future, building on Corollary 1.10 .

The paper is organized as follows. In Section 2, we set up the notation and establish some preliminary results. We prove the key Theorem 1.1, as well as Corollary 1.6, in Section 3. In Section 4, we construct a local system $\mathcal{Z}_{\left(w_{1}, w_{2}\right)}^{F}$ on the $h$-fold product of the Lagrangian and its free loop space, which traces the twisting coming from the righthand side in (1-2). We then show that its pullback is canonically isomorphic to the local system twisted by the first Stiefel-Whitney class of $\operatorname{det}\left(D_{(E, F)}\right)$, thus establishing Theorem 1.7. Corollaries 1.8 and 1.10 are proved at the end of the section.

Acknowledgements The present paper is based on a portion of the author's thesis work completed at Stanford University. The author would like to thank her advisor Eleny Ionel for her guidance and encouragement throughout the years. The author would also like to thank Aleksey Zinger for suggesting Corollary 1.10 and his help with the exposition and the referees for a comprehensive evaluation of this work and valuable comments.

This work was partially supported by NSF grants DMS-0605003 and DMS-0905738.

\section{Conventions and preliminaries}

Let $X, Y$ be Banach spaces and $D: X \rightarrow Y$ be a Fredholm operator. The determinant line of $D$ is defined as

$$
\operatorname{det}(D):=\bigwedge^{\text {top }} \operatorname{Ker}(D) \otimes \bigwedge^{\text {top }} \operatorname{Coker}(D)^{\vee}
$$


A short exact sequence of Fredholm operators

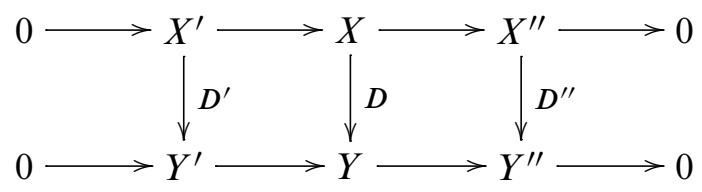

determines a canonical isomorphism

$$
\operatorname{det}(D) \cong \operatorname{det}\left(D^{\prime}\right) \otimes \operatorname{det}\left(D^{\prime \prime}\right) .
$$

For a continuous family of Fredholm operators $D_{t}: X_{t} \rightarrow Y_{t}$ parameterized by a topological space $B$, the determinant lines $\operatorname{det}\left(D_{t}\right)$ form a line bundle over $B$; see [10, Section A.2]. For a short exact sequence of such families, the isomorphisms (2-1) give rise to a canonical isomorphism between determinant line bundles.

Let $\Sigma$ be a nodal bordered Riemann surface and $\pi: \widetilde{\Sigma} \rightarrow \Sigma$ be its normalization; fix an ordering of the nodes of $\Sigma$ and the boundary components of $\widetilde{\Sigma}$. A real CauchyRiemann operator $D_{(E, F)}$ on $(E, F) \rightarrow \Sigma$ corresponds to a real Cauchy-Riemann operator $\widetilde{D}_{(E, F)}=\bigoplus_{i} D^{i}$ on $(\widetilde{E}, \widetilde{F}) \equiv \pi^{*}(E, F) \rightarrow \widetilde{\Sigma}$, where the sum is taken over the components of $\widetilde{\Sigma}$. Thus, by (2-1), there is a canonical isomorphism

$$
\operatorname{det}\left(\tilde{D}_{(E, F)}\right) \cong \bigotimes_{i} \operatorname{det}\left(D^{i}\right)
$$

On the other hand, by gluing together punctured disks around the special points in $\widetilde{\Sigma}$, we obtain a smooth surface $\Sigma^{\varepsilon}$ and a real Cauchy-Riemann operator $D^{\varepsilon}$ over $\Sigma^{\varepsilon}$ for a gluing parameter $\varepsilon$. By [2, Section 3.2] and [18, Section 4.1], for every sufficiently small $\varepsilon$ there is a canonical isomorphism

$$
\operatorname{det}\left(D^{\varepsilon}\right) \cong \operatorname{det}\left(\tilde{D}_{(E, F)}\right) \otimes \bigwedge^{\mathrm{top}}\left(\bigoplus_{j} E_{z_{j}} \oplus \bigoplus_{j} F_{x_{j}}\right)^{\vee},
$$

where $z_{j}$ and $x_{j}$ are the interior and boundary nodes, respectively. Moreover, the gluing maps satisfy an associativity property: the isomorphism (2-2) is independent of the order in which we smooth the nodes.

Remark 2.1 Let $(E, F) \rightarrow(\Sigma, \partial \Sigma)$ be a bundle pair. Choose a trivialization of $E$ over a curve $C_{\epsilon} \subset \Sigma$ isotopic to one of the boundary components of $\Sigma$. This trivialization can be extended over a neighborhood $U$ of the curve $C_{\epsilon}$. Pinching $\Sigma$ along $C_{\epsilon}$, we obtain a nodal curve $\Sigma^{S}$ with a diffeomorphism $\left(\Sigma-C_{\epsilon}\right) \rightarrow\left(\Sigma^{S}\right.$-node $)$. We can pull back the bundle pair $(E, F)$ to $\left(\Sigma^{S}\right.$-node). The trivialization of $E$ over the neighborhood $U$ of the curve $C_{\epsilon}$ induces a trivialization in a punctured neighborhood 
of the node. It can be uniquely extended over $\Sigma^{s}$. We say that the bundle pair $(E, F)$ descends to a bundle pair on the nodal surface.

Lemma 2.2 Let $(\Sigma, \partial \Sigma)$ be a smooth oriented surface with boundary. Every diffeomorphism $h:(\Sigma, \partial \Sigma) \rightarrow(\Sigma, \partial \Sigma)$ which preserves the orientation and each boundary component is isotopic to a diffeomorphism which restricts to the identity on a neighborhood of $\partial \Sigma$ in $\Sigma$.

Proof Fix a component $(\partial \Sigma)_{i} \cong S^{1}$ of $\partial \Sigma$, an identification of a neighborhood of $(\partial \Sigma)_{i}$ in $\Sigma$ with $S^{1} \times[0,2 \delta]$, and $\epsilon \in(0, \delta)$ such that $h\left(S^{1} \times[0, \epsilon]\right) \subset S^{1} \times[0,2 \delta]$. After composing $h$ with a path of diffeomorphisms on $\Sigma$ which restrict to the identity outside $S^{1} \times[0,2 \delta]$, we can assume that $h\left(S^{1} \times[0, \epsilon]\right)=S^{1} \times[0, \epsilon]$. By Farb and Margalit [3, Proposition 2.4] and [9, (1.1)], the group of diffeomorphisms of the cylinder preserving the orientation and each boundary component is path-connected. Thus, there is a path of diffeomorphisms

$$
f_{t}: S^{1} \times[0, \epsilon] \rightarrow S^{1} \times[0, \epsilon] \text { such that } f_{0}=\mathrm{id}, f_{1}=h_{\mid S^{1} \times[0, \epsilon]}^{-1} .
$$

The path $f_{t}$ generates a time-dependent vector field $X_{t}$. By multiplying $X_{t}$ by a bump function on $\Sigma$ vanishing outside $[0, \epsilon]$ and restricting to 1 on $S^{1} \times\left[0, \frac{\epsilon}{2}\right]$, we obtain a time-dependent vector field $\widetilde{X}_{t}$ on $\Sigma$. This new vector field gives rise to diffeomorphisms $\tilde{f}_{t}$ of $\Sigma$ which are identity outside $S^{1} \times[0, \epsilon]$, while $\tilde{f}_{1}$ restricts to $h^{-1}$ on $S^{1} \times\left[0, \frac{\epsilon}{2}\right]$. Then $h \circ \tilde{f}_{t}$ is a path of diffeomorphisms connecting $h$ with a diffeomorphism which restricts to the identity in a neighborhood of $(\partial \Sigma)_{i}$.

Lemma 2.3 Let $(\Sigma, \partial \Sigma)$ be a smooth oriented surface with boundary and $\phi \in \mathcal{D}$. Every family of real Cauchy-Riemann operators on a bundle pair $(E, F)$ over $M_{\phi}$ can be smoothly deformed to a family of real Cauchy-Riemann operators on a bundle pair $\left(E^{\prime}, F^{\prime}\right)$ over $M_{\phi^{\prime}}$ for some $\phi^{\prime} \in \mathcal{D}$ such that $\phi^{\prime}$ restricts to the identity on a neighborhood of $\partial \Sigma$.

Proof By Lemma 2.2, there exists a path $h_{s}$ in $\mathcal{D}$ such that $h_{0}=\phi$ and $h_{1}$ restricts to the identity on a neighborhood of $\partial \Sigma$ in $\Sigma$. Set $f_{s}=\phi^{-1} \circ h_{s}$. Let $\left(j_{t}, E_{t}, F_{t}, D_{t}\right)$, with $t \in I$, be any family of tuples such that $j_{t}$ is a complex structure on $\Sigma, D_{t}$ is a real Cauchy-Riemann operator on $\left(E_{t}, F_{t}\right)$ over $(\Sigma, \partial \Sigma)$, and

$$
\left(j_{1}, E_{1}, F_{1}, D_{1}\right)=\left(\phi^{*} j_{0}, \phi^{*} E_{0}, \phi^{*} F_{0}, \phi^{*} D_{0}\right) .
$$

For each $s \in I$, let

$$
\left(j_{s ; t}, E_{s ; t}, F_{s ; t}, D_{s ; t}\right)=\left(f_{s t}^{*} j_{t}, f_{s t}^{*} E_{t}, f_{s t}^{*} F_{t}, f_{s t}^{*} D_{t}\right) .
$$


Since $\left(j_{s ; 1}, E_{s ; 1}, F_{s ; 1}, D_{s ; 1}\right)=\left(h_{s}^{*} j_{s ; 0}, h_{s}^{*} E_{s ; 0}, h_{s}^{*} F_{s ; 0}, h_{s}^{*} D_{s ; 0}\right)$, this defines families of real Cauchy-Riemann operators on the bundle pairs $\left(E_{s}, F_{s}\right)$ over $M_{h_{s}}$. Since $h_{0}=\phi$, we have thus constructed the desired deformation of the original family.

Lemma 2.4 Every loop $\gamma$ in $\mathcal{H}_{l, \boldsymbol{k}}^{g, h}(M, L, \boldsymbol{b})$ lifts to a path $\widetilde{\gamma}$ in $\mathfrak{B}_{l, \boldsymbol{k}}^{g, h}(M, L, \boldsymbol{b}) \times \mathcal{J}_{\Sigma}$ such that $\widetilde{\gamma}_{1}=\phi \cdot \widetilde{\gamma}_{0}$ for some $\phi \in \mathcal{D}$ with $\phi_{\mid \partial \Sigma}=\mathrm{id}$.

Proof Under the assumption of Remark 1.4, the projection

$$
\mathfrak{B}_{l, \boldsymbol{k}}^{g, h}(M, L, \boldsymbol{b}) \times \mathcal{J}_{\Sigma} \rightarrow \mathcal{H}_{l, \boldsymbol{k}}^{g, h}(M, L, \boldsymbol{b})
$$

admits local slices. Thus, there exists a path $\widetilde{\gamma}_{t}=\left(u_{t}, j_{t}\right)$ in $\mathfrak{B}_{l, \boldsymbol{k}}^{g, h}(M, L, \boldsymbol{b}) \times \mathcal{J}_{\Sigma}$ lifting $\gamma$. Let $\phi \in \mathcal{D}$ be such that $\widetilde{\gamma}_{1}=\phi \cdot \widetilde{\gamma}_{0}$. By Lemma 2.2, there exists a path $h_{t}$ in $\mathcal{D}$ such that $h_{0}=\phi$ and $h_{1}$ restricts to the identity on the boundary. The lift $\widetilde{\gamma}_{t}^{\prime}=h_{t} \cdot \phi^{-1} \cdot \widetilde{\gamma}_{t}$ of $\gamma$ then satisfies $\widetilde{\gamma}_{1}^{\prime}=h_{1} \cdot \widetilde{\gamma}_{0}^{\prime}$.

\section{Determinant line bundles over loops}

We begin this section by deducing Theorem 1.1 from Propositions 3.1 and 3.2 below, which treat two distinct cases of Theorem 1.1. We then verify each of the two propositions for the trivial mapping cylinder over the disk with an additional assumption on the Maslov index of the pair $(E, F)$ on each fiber; see Lemmas 3.4 and 3.6. The full statements of Propositions 3.1 and 3.2 are then reduced to Lemmas 3.4 and 3.6, respectively. We conclude this section by proving Corollary 1.6.

Proposition 3.1 Let $\Sigma$ be a smooth oriented bordered surface, $\phi: \Sigma \rightarrow \Sigma$ be a diffeomorphism preserving the orientation and each boundary component, and $(E, F)$ be a bundle pair over $\left(M_{\phi}, \partial M_{\phi}\right)$ with $F$ orientable. If $D$ is any family of real Cauchy-Riemann operators on $(E, F)$, then

$$
\left\langle w_{1}(\operatorname{det} D), S^{1}\right\rangle=\sum_{i}\left\langle w_{2}(F),\left(\partial M_{\phi}\right)_{i}\right\rangle .
$$

Proposition 3.2 Let $\Sigma$ be a smooth oriented bordered surface, $\phi: \Sigma \rightarrow \Sigma$ be a diffeomorphism preserving the orientation and each boundary component, and $(E, F)$ be a bundle pair over $\left(M_{\phi}, \partial M_{\phi}\right)$ with $\mathrm{rk} F=1$. For each boundary component $(\partial \Sigma)_{i}$ of $\Sigma$, choose a section $\alpha_{i}$ of

$$
\left(\partial M_{\phi}\right)_{i}=M_{\phi_{\mid(\partial \Sigma)_{i}}} \rightarrow S^{1} .
$$

If $D$ is any family of real Cauchy-Riemann operators on $(E, F)$, then

$$
\left\langle w_{1}(\operatorname{det} D), S^{1}\right\rangle=\sum_{i}\left(\left\langle w_{1}(F),(\partial \Sigma)_{i}\right\rangle+1\right)\left\langle w_{1}(F), \alpha_{i}\right\rangle
$$


Remark 3.3 The space of real Cauchy-Riemann operators $(E, F) \rightarrow(\Sigma, \partial \Sigma)$ is contractible; thus, a choice of orientation on one determinant line canonically induces orientations on the rest. Moreover, any two families of real Cauchy-Riemann operators on a family $\left(E_{t}, F_{t}\right) \rightarrow\left(\Sigma_{t}, \partial \Sigma_{t}\right)$ are fiberwise homotopic. This implies that their determinant bundles have the same Stiefel-Whitney class.

Proof of Theorem 1.1 By Remark 3.3, it is sufficient to prove the result for some family of real Cauchy-Riemann operators on $(E, F) \rightarrow\left(M_{\phi}, \partial M_{\phi}\right)$. A connection on $E$ induces a family of complex linear Cauchy-Riemann operators $\bar{\partial}_{(E, F)}$ over $M_{\phi}$, which on a fiber $\Sigma_{t}$ is given by the complex linear Cauchy-Riemann operator of the restricted connection. Let

$$
\left(E^{1}, F^{1}\right)=\left(\operatorname{det}_{\mathbb{C}} E, \operatorname{det} F\right), \quad(\widetilde{E}, \widetilde{F})=\left(E \oplus 3 E^{1}, F \oplus 3 F^{1}\right) .
$$

The connection on $E$ induces connections on $E^{1}, \widetilde{E}$, and $4 E^{1}$ and thus families of complex linear Cauchy-Riemann operators $\bar{\partial}_{(\widetilde{E}, \widetilde{F})}, \bar{\partial}_{\left(E^{1}, F^{1}\right)}$, and $\bar{\partial}_{\left(4 E^{1}, 4 F^{1}\right)}$. By $(2-1)$,

$$
\begin{aligned}
\operatorname{det}\left(\bar{\partial}_{(\widetilde{E}, \widetilde{F})}\right) \otimes \operatorname{det}\left(\bar{\partial}_{\left(E^{1}, F^{1}\right)}\right) & \cong \operatorname{det}\left(\bar{\partial}_{\left(\widetilde{E} \oplus E^{1}, \widetilde{F} \oplus F^{1}\right)}\right) \\
& =\operatorname{det}\left(\bar{\partial}_{\left(E \oplus 4 E^{1}, F \oplus 4 F^{1}\right)}\right) \\
& \cong \operatorname{det}\left(\bar{\partial}_{(E, F)}\right) \otimes \operatorname{det}\left(\bar{\partial}_{\left(4 E^{1}, 4 F^{1}\right)}\right)
\end{aligned}
$$

Therefore,

$$
\begin{aligned}
w_{1}(\operatorname{det}(D)) & =w_{1}\left(\operatorname{det}\left(\bar{\partial}_{(E, F)}\right)\right) \\
& =w_{1}\left(\operatorname{det}\left(\bar{\partial}_{(\widetilde{E}, \widetilde{F})}\right)\right)+w_{1}\left(\operatorname{det}\left(\bar{\partial}_{\left(E^{1}, F^{1}\right)}\right)\right)+w_{1}\left(\operatorname{det}\left(\bar{\partial}_{\left(4 E^{1}, 4 F^{1}\right)}\right)\right) .
\end{aligned}
$$

By Proposition 3.1,

$$
\begin{aligned}
\left\langle w_{1}\left(\operatorname{det}\left(\bar{\partial}_{(\widetilde{E}, \widetilde{F})}\right)\right), S^{1}\right\rangle & =\sum_{i}\left\langle w_{2}(\widetilde{F}),\left(\partial M_{\phi}\right)_{i}\right\rangle=\sum_{i}\left\langle w_{2}(F),\left(\partial M_{\phi}\right)_{i}\right\rangle, \\
\left\langle w_{1}\left(\operatorname{det}\left(\bar{\partial}_{\left(4 E^{1}, 4 F^{1}\right)}\right)\right), S^{1}\right\rangle & =\sum_{i}\left\langle w_{2}\left(4 F^{1}\right),\left(\partial M_{\phi}\right)_{i}\right\rangle=0 .
\end{aligned}
$$

By Proposition 3.2,

$$
\begin{aligned}
\left\langlew _ { 1 } \left(\operatorname { d e t } \left(\bar{\partial}_{\left.\left.\left.\left(E^{1}, F^{1}\right)\right)\right), S^{1}\right\rangle}\right.\right.\right. & =\sum_{i}\left(\left\langle w_{1}\left(F^{1}\right),(\partial \Sigma)_{i}\right\rangle+1\right)\left\langle w_{1}\left(F^{1}\right), \alpha_{i}\right\rangle \\
& =\sum_{i}\left(\left\langle w_{1}(F),(\partial \Sigma)_{i}\right\rangle+1\right)\left\langle w_{1}(F), \alpha_{i}\right\rangle
\end{aligned}
$$

Combining the last four identities, we obtain Theorem 1.1. 
Lemma 3.4 Let $(E, F) \rightarrow\left(D^{2}, \partial D^{2}\right) \times S^{1}$ be a bundle pair with $F$ orientable and Maslov index zero on each fiber. If $D$ is a family of real Cauchy-Riemann operators on $(E, F)$, then

$$
\left\langle w_{1}(\operatorname{det} D), S^{1}\right\rangle=\left\langle w_{2}(F), \partial D^{2} \times S^{1}\right\rangle .
$$

Proof The standard $\bar{\partial}_{0}$-operator on $\left(\mathbb{C}^{n}, \mathbb{R}^{n}\right) \rightarrow\left(D^{2}, \partial D^{2}\right)$ is surjective and its kernel consists of constant real-valued sections; see [10, Theorem C.1.10]. If the bundle pair $(E, F) \rightarrow\left(D^{2}, \partial D^{2}\right) \times S^{1}$ is trivializable, we can consider the constant family of standard $\bar{\partial}_{0}$-operators on a trivialization

$$
(E, F) \cong\left(\mathbb{C}^{n} \times D^{2}, \mathbb{R}^{n} \times \partial D^{2}\right) \times S^{1} .
$$

The determinant bundle of this family is isomorphic to $\mathbb{R}^{n} \times S^{1}$ by evaluation at a boundary point and in particular is orientable. By Remark 3.3, the determinant bundle of the family $D$ is also orientable.

If $\left(E^{\prime}, F^{\prime}\right) \rightarrow\left(D^{2}, \partial D^{2}\right) \times S^{1}$ is another bundle pair,

$\operatorname{det} D_{(E, F)} \otimes \operatorname{det} D_{\left(E^{\prime}, F^{\prime}\right)} \cong \operatorname{det}\left(D_{(E, F)} \oplus D_{\left(E^{\prime}, F^{\prime}\right)}\right) \cong \operatorname{det} D_{\left(E \oplus E^{\prime}, F \oplus F^{\prime}\right)}$

by (2-1). Thus, we can stabilize $(E, F)$ with a trivial bundle pair and assume that $n=\operatorname{rk} F>2$. Since $\pi_{1}(\mathrm{SO}(n)) \cong \mathbb{Z}_{2}$ and the homomorphism $\pi_{1}(\mathrm{SO}(n)) \rightarrow \pi_{1}(U(n))$ induced by the inclusion is trivial, the second Stiefel-Whitney class $w_{2}(F)$ then classifies the bundle pairs $(E, F)$ over $\left(D^{2}, \partial D^{2}\right) \times S^{1}$. Thus, if $w_{2}(F)=0$, the bundle pair $(E, F)$ is trivializable and the determinant bundle $\operatorname{det}(D)$ is orientable. If $w_{2}(F) \neq 0$, the bundle pair $(E, F)$ is isomorphic to a stabilization of the bundle pair in [4, Proposition 8.1.7], which constructs a nonorientable family of real Cauchy-Riemann operators. Combining the two cases gives the result.

Lemma 3.5 If $(E, F) \rightarrow\left(D^{2}, \partial D^{2}\right)$ is a bundle pair with $\mathrm{rk}_{\mathbb{C}} E=1$ and Maslov index $\mu=\mu(E, F) \geq-1$, every real Cauchy-Riemann operator $D$ on $(E, F)$ is surjective. Moreover, if $x_{1}, \ldots, x_{\mu+1} \in \partial D^{2}$ are distinct points, then the homomorphism

$$
\mathrm{ev}: \operatorname{Ker}(D) \rightarrow \bigoplus_{i=1}^{\mu+1} F_{x_{i}}, \quad \operatorname{ev}(\xi)=\left(\xi\left(x_{1}\right), \ldots, \xi\left(x_{\mu+1}\right)\right),
$$

is an isomorphism.

Proof By [10, Theorem C.3.5 and Corollary C.3.9], the bundle pair $(E, F)$ is isomorphic to $\left(\mathbb{C} \times D^{2}, \Lambda\right)$, where the fiber at $e^{i \theta} \in \partial D^{2} \cong S^{1}$ is given by

$$
\Lambda_{e^{\mathrm{i} \theta}}=e^{\frac{\mathrm{i} \theta \mu}{2}} \mathbb{R} .
$$


By [10, Theorem C.1.10], the standard Cauchy-Riemann operator $\bar{\partial}_{0}$ on $\left(\mathbb{C} \times D^{2}, \Lambda\right)$ is surjective if $\mu \geq-1$ and thus $\operatorname{dim} \operatorname{Ker} \bar{\partial}_{0}=\mu+1$. Moreover, the elements of the kernel are polynomials $\xi(z)=a_{0}+\cdots+a_{\mu} z^{\mu}$ with $a_{i}=\bar{a}_{\mu-i}$. The kernel of the homomorphism

$$
\mathrm{ev}: \operatorname{Ker}\left(\bar{\partial}_{0}\right) \rightarrow \bigoplus_{i=1}^{\mu+1} \Lambda_{x_{i}}, \quad \operatorname{ev}(\xi)=\left(\xi\left(x_{1}\right), \ldots, \xi\left(x_{\mu+1}\right)\right),
$$

consists of polynomials (of degree $\mu$ ) which vanish at the $\mu+1$ points $x_{i}$; therefore, this homomorphism is injective. Since the domain and target are of the same dimension, the homomorphism (3-2) is an isomorphism.

Let $D^{\prime}$ be any real Cauchy-Riemann operator on the above bundle pair $\left(\mathbb{C} \times D^{2}, \Lambda\right)$. By [10, Theorem C.1.10], $D^{\prime}$ is still surjective and $\operatorname{dim} \operatorname{Ker}\left(D^{\prime}\right)=\mu+1$. If the homomorphism (3-2) with $\bar{\partial}_{0}$ replaced by $D^{\prime}$ is not an isomorphism, there exists $\xi \in \operatorname{Ker}\left(D^{\prime}\right)-\{0\}$ vanishing at the $\mu+1$ points $x_{i}$. By [10, Section C.4], there exists $f:\left(D^{2}, \partial D^{2}\right) \rightarrow\left(\mathbb{C}^{*}, \mathbb{R}^{*}\right)$ such that $\bar{\partial}_{0}(f \xi)=0$. Since $f \xi$ vanishes at the $\mu+1$ points, by the previous paragraph $\xi$ is identically zero. Thus, the homomorphism (3-2) with $\bar{\partial}_{0}$ replaced by $D^{\prime}$ is in fact an isomorphism.

An isomorphism $\varphi:(E, F) \rightarrow\left(\mathbb{C} \times D^{2}, \Lambda\right)$ induces a commutative diagram

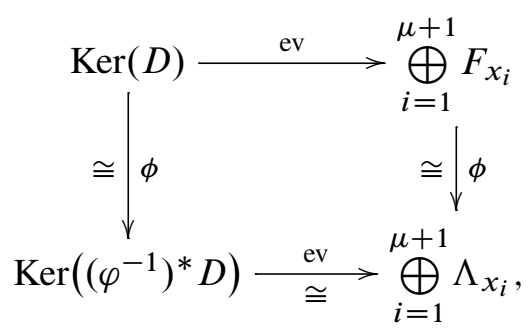

where $\left(\varphi^{-1}\right)^{*} D$ is the induced real Cauchy-Riemann operator on $\left(\mathbb{C} \times D^{2}, \Lambda\right)$. Since three of the maps in the diagram are isomorphisms, so is the evaluation map (3-1).

Lemma 3.6 Let $(E, F) \rightarrow\left(D^{2}, \partial D^{2}\right) \times S^{1}$ be a bundle pair with rk $F=1$ and a nonnegative Maslov index $\mu$. If $D$ is a family of real Cauchy-Riemann operators on $(E, F)$ and $x \in \partial D^{2}$, then

$$
\left\langle w_{1}(\operatorname{det} D), S^{1}\right\rangle=\left(\left\langle w_{1}(F), \partial D^{2}\right\rangle+1\right)\left\langle w_{1}(F), x \times S^{1}\right\rangle .
$$

Proof By Lemma 3.5, the operators $D_{t}, t \in S^{1}$, are surjective and

$$
\text { ev: } \operatorname{Ker}\left(D_{t}\right) \rightarrow \bigoplus_{i=1}^{\mu+1} F_{\mid x_{i} \times t}, \quad \operatorname{ev}(\xi)=\left(\xi\left(x_{1}\right), \ldots, \xi\left(x_{\mu+1}\right)\right),
$$


are isomorphisms for any choice of distinct points $x_{1}, \ldots, x_{\mu+1} \in \partial D^{2}$. Therefore,

$$
\begin{aligned}
\left\langle w_{1}(\operatorname{det}(D)), S^{1}\right\rangle=\left\langle w_{1}\left(\bigoplus_{i=1}^{\mu+1} F_{\mid x_{i} \times t}\right), S^{1}\right\rangle & \left.=\sum_{i=1}^{\mu+1}\left\langle w_{1}(F), x_{i} \times S^{1}\right)\right\rangle \\
& =(\mu+1)\left\langle w_{1}(F), x_{1} \times S^{1}\right\rangle .
\end{aligned}
$$

Since the Maslov index $\mu$ modulo two is $\left\langle w_{1}(F), \partial D^{2}\right\rangle$,

$$
(\mu+1)\left\langle w_{1}(F), x_{1} \times S^{1}\right\rangle=\left(\left\langle w_{1}(F), \partial D^{2}\right\rangle+1\right)\left\langle w_{1}(F), x_{1} \times S^{1}\right\rangle,
$$

establishing the formula.

Proof of Proposition 3.1 By Lemma 2.3, we can assume that $\phi$ restricts to the identity in a neighborhood of the boundary. For each boundary component $(\partial \Sigma)_{i}$ of $\Sigma$, let

$$
U_{i} \cong[0,2 \epsilon] \times(\partial \Sigma)_{i} \times S^{1} \equiv \mathrm{Cyl} \times S^{1}
$$

be a neighborhood of $(\partial \Sigma)_{i} \times S^{1}$ in $M_{\phi}$. Let $j_{0}$ be a standard complex structure on $[0,2 \epsilon] \times(\partial \Sigma)_{i}$. Since every loop $\gamma$ of complex structures on the cylinder Cyl is of the form $j_{t}=\psi_{t}^{*} j_{0}$ for some loop of diffeomorphisms $\psi_{t}$, there is an isomorphism

$$
\left(\mathrm{Cyl} \times S^{1} ; \gamma\right) \cong\left(\operatorname{Cyl} \times S^{1} ; j_{0}\right), \quad\left(x, t ; j_{t}\right) \mapsto\left(\psi_{t}(x), t ;\left(\psi_{t}^{-1}\right)^{*} j_{t}=j_{0}\right) .
$$

Thus, there is an isomorphism

$$
\left(U_{i}, j_{t \mid U_{i}}\right) \cong\left([0,2 \epsilon] \times(\partial \Sigma)_{i} \times S^{1}, j_{0}\right) .
$$

Let $I=[0,1]$ as before. For each $\delta \in[0,2 \epsilon]$, let

$$
\tilde{U}_{i}(\delta) \cong[0, \delta] \times(\partial \Sigma)_{i} \times I
$$

be the neighborhood of $(\partial \Sigma)_{i} \times I \subset \Sigma \times I$ corresponding to $[0, \delta] \times(\partial \Sigma)_{i} \times S^{1}$ under the identification (3-3); for example, $\tilde{U}_{i}(0) \cong\{0\} \times(\partial \Sigma)_{i} \times I$. We can trivialize $F$ over $\tilde{U}_{i}(0)$, since $F$ is orientable and $\tilde{U}_{i}(0)$ is homotopic to a circle. A trivialization $F_{\mid} \tilde{U}_{i}(0) \cong \mathbb{R}^{n} \times \tilde{U}_{i}(0)$ induces a trivialization

$$
E_{\mid \tilde{U}_{i}(0)} \cong F \oplus J F_{\mid \widetilde{U}_{i}(0)} \cong \mathbb{C}^{n} \times \tilde{U}_{i}(0),
$$

which we can extend to a trivialization $E_{\mid \tilde{U}_{i}(2 \epsilon)} \cong \mathbb{C}^{n} \times \tilde{U}_{i}(2 \epsilon)$.

At the two endpoints of the interval $I$, glue the trivial bundles $\mathbb{R}^{n} \times(\partial \Sigma)_{i} \times 1$ and $\mathbb{R}^{n} \times(\partial \Sigma)_{i} \times 0$ using a clutching map $g_{i}:(\partial \Sigma)_{i} \rightarrow \mathrm{SO}(n)$ so that the bundle pair

$$
\left(\mathbb{C}^{n} \times(\partial \Sigma)_{i}, \mathbb{R}^{n} \times(\partial \Sigma)_{i}\right) \times\left(g_{i}, g_{i}\right) I \rightarrow(\partial \Sigma)_{i} \times S^{1}
$$




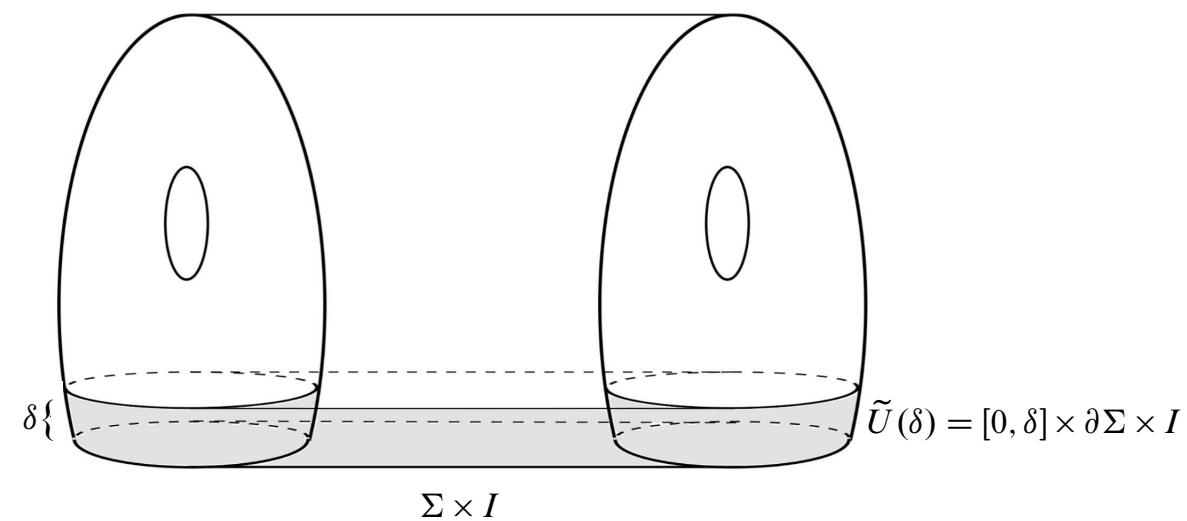

is isomorphic to $\left(E_{\mid(\partial \Sigma)_{i} \times S^{1}}, F_{\mid(\partial \Sigma)_{i} \times S^{1}}\right)$. Since the inclusion $\mathrm{SO}(n) \rightarrow U(n)$ is nullhomotopic, the map $g_{i}$ can be extended to a map

$$
\tilde{g}_{i}:[0,2 \epsilon] \times(\partial \Sigma)_{i} \rightarrow U(n) \quad \text { such that } \tilde{g}_{i \mid\left[\frac{\epsilon}{2}, 2 \epsilon\right]}=\mathrm{id} .
$$

For every $t \in S^{1}$, pinch $\Sigma \times t$ along the curve $\epsilon \times(\partial \Sigma)_{i} \times t$ to obtain a nodal curve $\Sigma^{s}$ with normalization consisting of a disjoint union of disks $D_{i}^{2}$ and a closed Riemann surface $\hat{\Sigma}$, with special points $0 \in D_{i}^{2}$ and $p_{i} \in \hat{\Sigma}$. The bundle pair $(E, F)$ descends to a bundle pair over the family of nodal curves as in Remark 2.1, inducing bundles

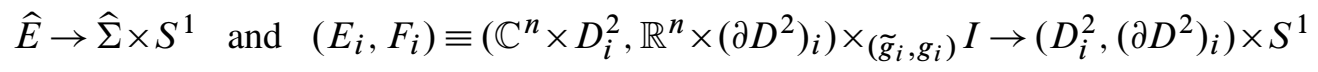

with isomorphisms $\widehat{E}_{\mid p_{i} \times t} \cong \mathbb{C}^{n} \cong E_{i \mid 0 \times t}$ for every $t \in S^{1}$.

We are interested in the first Stiefel-Whitney class of the family of real CauchyRiemann operators $D_{(E, F)}$. Taking a family of complex linear Cauchy-Riemann operators $\hat{D}$ on $\widehat{E}$ and gluing it to a family of real Cauchy-Riemann operators $D_{i}$ on $\left(E_{i}, F_{i}\right)$, we obtain a family of real Cauchy-Riemann operators $D^{\varepsilon}$ on $(E, F)$. By Remark 3.3 and (2-2),

$$
\operatorname{det}\left(D_{(E, F)}\right) \cong \operatorname{det}\left(D^{\varepsilon}\right) \cong \operatorname{det}(\widehat{D}) \otimes \bigotimes_{i}\left(\operatorname{det}\left(D_{i}\right) \otimes \bigwedge^{\mathrm{top}}\left(\widehat{E}_{\mid\left(p_{i}, t\right)}\right)\right)
$$

and thus

$$
w_{1}(\operatorname{det}(D))=w_{1}(\operatorname{det}(\hat{D}))+\sum_{i}\left(w_{1}\left(\operatorname{det}\left(D_{i}\right)\right)+w_{1}\left(\widehat{E}_{p_{i} \times S^{1}}\right)\right) .
$$

The complex structure on the kernels and cokernels of the family of operators $\widehat{D}$ induces a canonical orientation on $\operatorname{det}(\hat{D})$; in particular, $w_{1}(\operatorname{det}(\hat{D}))$ is zero. Moreover, $\widehat{E}_{p_{i} \times S_{1}} \cong \mathbb{C}^{n} \times S^{1}$ also has a canonical orientation and $w_{1}\left(\widehat{E}_{p_{i} \times S_{1}}\right)$ is zero. Therefore, 
the problem reduces to the families of operators $D_{i}$ on $\left(E_{i}, F_{i}\right)$ over $\left(D_{i}^{2},\left(\partial D_{i}^{2}\right)\right) \times S^{1}$. Lemma 3.4 now gives the result.

Proof of Proposition 3.2 For each boundary component $(\partial \Sigma)_{i}$ of $\Sigma$, let $\tilde{U}_{i}(\delta)$ be as in the proof of Proposition 3.1. Let $m_{i} \in\{0,1\}$ be equal to 0 if $\left\langle w_{1}(F),(\partial \Sigma)_{i}\right\rangle=0$ and 1 if $\left\langle w_{1}(F),(\partial \Sigma)_{i}\right\rangle=1$. Then there is an isomorphism

$$
(E, F)_{\mid \widetilde{U}_{i}(0)} \cong\left(\mathbb{C} \times \widetilde{U}_{i}(0), \Lambda_{i} \times I\right),
$$

where the fiber of $\Lambda_{i}$ at a point $e^{\mathrm{i} \theta} \times t \in(\partial \Sigma)_{i} \times I$ is given by $e^{\mathrm{i} \theta m_{i} / 2} \mathbb{R} \subset \mathbb{C}$. We can extend the trivialization $E_{\mid \widetilde{U}_{i}(0)} \cong \mathbb{C} \times \widetilde{U}_{i}(0)$ to the neighborhood $\widetilde{U}_{i}(2 \epsilon)$.

At the two endpoints of the interval $I$, glue the bundles $\Lambda_{i} \times 1$ and $\Lambda_{i} \times 0$ using a clutching map $g_{i}:(\partial \Sigma)_{i} \rightarrow\{ \pm 1\}$ so that the bundle pair

$$
\left(\mathbb{C} \times(\partial \Sigma)_{i}, \Lambda_{i}\right) \times_{\left(g_{i}, g_{i}\right)} I \rightarrow(\partial \Sigma)_{i} \times S^{1}
$$

is isomorphic to $\left(E_{\mid(\partial \Sigma)_{i} \times S^{1}}, F_{\mid(\partial \Sigma)_{i} \times S^{1}}\right)$. Since the inclusion $O(1) \rightarrow U(1)$ is nullhomotopic, the map $g_{i}$ can be extended to a map

$$
\tilde{g}_{i}:[0,2 \epsilon] \times(\partial \Sigma)_{i} \rightarrow U(1) \quad \text { such that } \tilde{g}_{i \mid\left[\frac{\epsilon}{2}, 2 \epsilon\right]}=\mathrm{id} .
$$

For every $t \in S^{1}$, pinch $\Sigma \times t$ along the curve $\epsilon \times(\partial \Sigma)_{i} \times t$ to obtain a nodal curve $\Sigma^{s}$ as in the proof of Proposition 3.1. The bundle pair $(E, F)$ descends to a bundle pair over the family of nodal curves as in Remark 2.1, inducing bundles

$$
\widehat{E} \rightarrow \widehat{\Sigma} \times S^{1} \quad \text { and } \quad\left(E_{i}, F_{i}\right) \equiv\left(\mathbb{C} \times D_{i}^{2}, \Lambda_{i}\right) \times_{\left(\widetilde{g}_{i}, g_{i}\right)} I \rightarrow\left(D_{i}^{2},\left(\partial D^{2}\right)_{i}\right) \times S^{1},
$$

with isomorphisms $\widehat{E}_{\mid p_{i} \times t} \cong \mathbb{C} \cong E_{i \mid 0 \times t}$ for every $t \in S^{1}$.

Taking a family of complex linear Cauchy-Riemann operators $\widehat{D}$ on $\widehat{E}$ and gluing it to a family of real Cauchy-Riemann operators $D_{i}$ on $\left(E_{i}, F_{i}\right)$, we obtain a family of real Cauchy-Riemann operators $D^{\varepsilon}$ on $(E, F)$. By Remark 3.3 and (2-2),

$$
\operatorname{det}\left(D_{(E, F)}\right) \cong \operatorname{det}\left(D^{\varepsilon}\right) \cong \operatorname{det}(\widehat{D}) \otimes \bigotimes_{i}\left(\operatorname{det}\left(D_{i}\right) \otimes \bigwedge^{\max }\left(\widehat{E}_{\mid\left(p_{i}, t\right)}\right)\right)
$$

and thus

$$
w_{1}(\operatorname{det}(D))=w_{1}(\operatorname{det}(\widehat{D}))+\sum_{i}\left(w_{1}\left(\operatorname{det}\left(D_{i}\right)\right)+w_{1}\left(\widehat{E}_{p_{i} \times S^{1}}\right)\right) .
$$

As in the proof of Proposition 3.1, $w_{1}(\operatorname{det}(\widehat{D}))$ and $w_{1}\left(\widehat{E}_{p_{i} \times S^{1}}\right)$ vanish. Thus, the problem reduces to the families of operators $D_{i}$ on $\left(E_{i}, F_{i}\right)$ over $\left(D_{i}^{2},\left(\partial D_{i}^{2}\right)\right) \times S^{1}$. Lemma 3.6 now gives the result. 
Proof of Corollary 1.6 Let $\beta_{i}$ be as in Corollary 1.5. The sum $\sum \beta_{i} \in H_{2}(L)$ is the boundary of the class $S \in H_{3}(M, L)$ obtained by following the image in $(M, L)$ of the whole surface along the loop $\gamma$, that is $\sum \beta_{i}=\partial S$. Let

$$
\cdots \longrightarrow H^{2}\left(M ; \mathbb{Z}_{2}\right) \stackrel{i^{*}}{\longrightarrow} H^{2}\left(L ; \mathbb{Z}_{2}\right) \stackrel{\delta}{\longrightarrow} H^{3}\left(M, L ; \mathbb{Z}_{2}\right) \longrightarrow \cdots
$$

be the exact sequence for the pair $(M, L)$. Since $w_{2}(F)+\sigma^{2}$ is in the image of $i^{*}$, $\delta\left(w_{2}(F)+\sigma^{2}\right)=0$. Since $\beta_{i}$ is the class of a torus, $\left\langle\sigma^{2}, \beta_{i}\right\rangle=0$. Thus,

$$
0=\left\langle\delta\left(w_{2}(F)+\sigma^{2}\right),[S]\right\rangle=\left\langle\delta\left(w_{2}(F)\right),[S]\right\rangle+\left\langle\sigma^{2}, \sum \beta_{i}\right\rangle=\left\langle w_{2}(F), \sum \beta_{i}\right\rangle .
$$

The formula (1-2) thus reduces to (1-3).

\section{Local systems of determinant line bundles}

In this section we recall the basics of local systems following Steenrod [16] and construct a local system $\mathcal{Z}_{\left(w_{1}, w_{2}\right)}^{F}$ over the $h$-fold product of the Lagrangian $L$ and its free loop space $\mathcal{L}(L) .{ }^{1}$ We then show its pullback is canonically isomorphic to the local system twisted by the first Stiefel-Whitney class of $\operatorname{det}\left(D_{(E, F)}\right)$. We conclude this section by establishing Corollaries 1.8 and 1.10.

Definition 4.1 A system of local groups $\mathcal{G}$ on a path-connected topological space $L$ consists of

- a group $G_{x}$ for every $x \in L$ and

- a group isomorphism $\alpha_{x y}: G_{x} \rightarrow G_{y}$ for every homotopy class $\alpha_{x y}$ of paths from $x$ to $y$

such that the composition $\beta_{y z} \circ \alpha_{x y}$ is the isomorphism corresponding to the path $\alpha_{x y} \beta_{y z}$.

Lemma 4.2 (Steenrod [16, Theorem 1]) Suppose $p_{0} \in L, G$ is a group and

$$
\psi: \pi_{1}\left(L, p_{0}\right) \rightarrow \operatorname{Aut}(G)
$$

is a group homomorphism. Then there is a unique system $\mathcal{G}=\left\{G_{x}\right\}$ of local groups on $L$ such that $G_{0}=G$ and the operations of $\pi_{1}\left(L, p_{0}\right)$ on $G_{0}$ are those determined by $\psi$.

\footnotetext{
${ }^{1}$ Recall that $h$ is the number of boundary components of $\Sigma$.
} 
Two local system $\mathcal{G}$ and $\mathcal{G}^{\prime}$ on $L$ are isomorphic if for every point $x \in L$ there is an isomorphism $h_{x}: G_{x} \cong G_{x}^{\prime}$ such that $h_{x}=\alpha_{x y}^{-1} h_{y} \alpha_{x y}$ for every path $\alpha_{x y}$ between $x$ and $y$. Equivalently, two local system are isomorphic if the groups $G$ and $G^{\prime}$ are isomorphic and the induced actions of $\pi_{1}\left(L, x_{0}\right)$ are the same. There are $\operatorname{Aut}(G)$ of such isomorphisms, and one is fixed by a choice of an isomorphism $G_{x_{0}} \cong G_{x_{0}}^{\prime}$.

\section{Definition 4.3 Let}

$$
f:\left(L_{1}, p_{1}\right) \rightarrow\left(L_{2}, p_{2}\right)
$$

be a continuous map between path-connected topological spaces and let $\mathcal{G}=\left\{G_{x}\right\}$ be the local system on $L_{2}$ induced by $\psi: \pi_{1}\left(L_{2}, p_{2}\right) \rightarrow \operatorname{Aut}(G)$. The pullback system $\mathcal{G}^{\prime}=f^{*} \mathcal{G}$ is the system of local groups induced by $\psi \circ f_{\#}: \pi_{1}\left(L_{1}, p_{1}\right) \rightarrow \operatorname{Aut}(G)$, where $f_{\#}: \pi_{1}\left(L_{1}, p_{1}\right) \rightarrow \pi_{1}\left(L_{2}, p_{2}\right)$.

Definition 4.4 The local system of orientations for a vector bundle $F \rightarrow L$ is the system induced by the homomorphism $\psi: \pi_{1}\left(L, p_{0}\right) \rightarrow \operatorname{Aut}(\mathbb{Z})=\mathbb{Z}_{2}$ assigning to $\alpha \in \pi_{1}\left(L, p_{0}\right)$ the value of $\left\langle w_{1}(F), \alpha\right\rangle$. We denote this system by $\mathcal{Z}_{w_{1}(F)}$.

Remark 4.5 If $L$ is a smooth manifold, the local system of orientations for $T L$, $\mathcal{Z}_{w_{1}(T L)}$, is called the system of twisted integer coefficients in [16].

Given a vector bundle $F \rightarrow L$, we now construct a local system $\mathcal{Z}_{\left(w_{1}, w_{2}\right)}^{F}$ on the $h$-fold product of the Lagrangian and its free loop space $\mathcal{L}(L)$, which traces the twisting coming from the righthand side of (1-2). We begin by constructing a system over every component of $L \times \mathcal{L}(L)$ and thus define a system over $L \times \mathcal{L}(L)$. We then pullback $h$ copies of it to the product $(L \times \mathcal{L}(L))^{h}$ via the projection maps. The system $\mathcal{Z}_{\left(w_{1}, w_{2}\right)}^{F}$ is defined as the tensor product of the pulled-back systems.

Let $p_{i} \times \gamma_{j}$ be a basepoint for a component $L_{i} \times \mathcal{L}(L)_{j} \subset L \times \mathcal{L}(L)$. Define a local system over $L_{i} \times \mathcal{L}(L)_{j}$ using the homomorphism

$$
\begin{gathered}
\psi: \pi_{1}\left(L_{i} \times \mathcal{L}(L)_{j}, p_{i} \times \gamma_{j}\right)=\pi_{1}\left(L_{i}, p_{i}\right) \times \pi_{1}\left(\mathcal{L}(L)_{j}, \gamma_{j}\right) \rightarrow \operatorname{Aut}(\mathbb{Z})=\mathbb{Z}_{2}, \\
(\alpha, \beta) \mapsto\left(\left\langle w_{1}(F), \gamma_{j}\right\rangle+1\right)\left\langle w_{1}(F), \alpha\right\rangle+\left\langle w_{2}(F), \beta\right\rangle .
\end{gathered}
$$

Thus, the system $\mathcal{Z}_{\left(w_{1}, w_{2}\right)}^{F}$ over a component of $(L \times \mathcal{L}(L))^{h}$ with a basepoint $(\vec{p}, \vec{\gamma})=$ $\left(p_{1}, \gamma_{1}, \ldots, p_{h}, \gamma_{h}\right)$ is given by the homomorphism

$$
\begin{aligned}
& \psi: \pi_{1}\left((L \times \mathcal{L}(L))^{h},(\vec{p}, \vec{\gamma})\right) \rightarrow \operatorname{Aut}(\mathbb{Z})=\mathbb{Z}_{2}, \\
& \left(\alpha_{1}, \beta_{1}, \ldots, \alpha_{h}, \beta_{h}\right) \mapsto \sum_{i=1}^{h}\left(\left\langle w_{1}(F), \gamma_{i}\right\rangle+1\right)\left\langle w_{1}(F), \alpha_{i}\right\rangle+\sum_{i=1}^{h}\left\langle w_{2}(F), \beta_{i}\right\rangle .
\end{aligned}
$$


We next construct a natural isomorphism between the local system $\mathcal{Z}_{w_{1}\left(\operatorname{det} D_{(E, F)}\right)}$ on $\mathcal{H}_{l, \boldsymbol{k}}^{g, h}(M, L, \boldsymbol{b})$ and a pullback/pushdown of $\mathcal{Z}_{\left(w_{1}, w_{2}\right)}^{F}$.

Proposition 4.6 Suppose there is at least one boundary marked point on each boundary component of $\Sigma$. Then there is a map ev: $\mathcal{H}_{l, \boldsymbol{k}}^{g, h}(M, L, \boldsymbol{b}) \rightarrow(L \times \mathcal{L}(L))^{h}$ such that for every bundle pair $(E, F) \rightarrow(M, L)$ the local system $\mathcal{Z}_{w_{1}\left(\operatorname{det}\left(D_{(E, F)}\right)\right)}$ is isomorphic to the pulled-back system $\mathrm{ev}^{*} \mathcal{Z}_{\left(w_{1}, w_{2}\right)}^{F}$.

Proof The map ev to the $i^{\text {th }} L$ factor is given by the evaluation at the first marked point on the $i^{\text {th }}$ boundary component. We now construct the map to the $\mathcal{L}(L)$ factors. Denote by $\mathcal{D}_{b}$ and $\mathcal{D}_{x_{1}}$ the groups of orientation-preserving diffeomorphisms of $\Sigma$ that restrict to the identity on $\partial \Sigma$ and fix a point $x_{1, i}$ on each component of $\partial \Sigma$, respectively. The group $\mathcal{D}_{b}$ is a normal subgroup of $\mathcal{D}_{x_{1}}$, and the quotient $\mathcal{D}_{x_{1}} / \mathcal{D}_{b}$ is contractible. Thus,

$$
\left(\mathfrak{B}_{l, \boldsymbol{k}}^{g, h}(M, L, \boldsymbol{b}) \times \mathcal{J}_{\Sigma}\right) / \mathcal{D}_{b} \rightarrow\left(\mathfrak{B}_{l, \boldsymbol{k}}^{g, h}(M, L, \boldsymbol{b}) \times \mathcal{J}_{\Sigma}\right) / \mathcal{D}_{x_{1}}
$$

has a contractible fiber and we can choose a section $s$. Any two such sections are homotopic. Since the elements of $\mathcal{D}_{b}$ fix the boundary of $\Sigma$ pointwise, there is a map

$$
e_{i}:\left(\mathfrak{B}_{l, \boldsymbol{k}}^{g, h}(M, L, \boldsymbol{b}) \times \mathcal{J}_{\Sigma}\right) / \mathcal{D}_{b} \rightarrow \mathcal{L}(L), \quad\left[u, z, \boldsymbol{x}_{\mathbf{1}}, \ldots, \boldsymbol{x}_{\boldsymbol{h}}\right] \mapsto u_{\mid(\partial \Sigma)_{i}} .
$$

The map to the $i^{\text {th }} \mathcal{L}(L)$ factor $\mathcal{H}_{l, \boldsymbol{k}}^{g, h}(M, L, \boldsymbol{b})$ is the composition

$$
\mathrm{ev}_{i}: \mathcal{H}_{l, \boldsymbol{k}}^{g, h}(M, L, \boldsymbol{b}) \hookrightarrow\left(\mathfrak{B}_{l, \boldsymbol{k}-\mathbf{1}}(\Sigma, \boldsymbol{b}) \times \mathcal{J}_{\Sigma}\right) / \mathcal{D}_{x_{1}} \stackrel{e_{i} \circ s}{\longrightarrow} \mathcal{L}(L) .
$$

We restrict ourselves to a particular connected component. Let $u_{0} \in \mathcal{H}_{l, \boldsymbol{k}}^{g, h}(M, L, \boldsymbol{b})$ map under ev to the basepoint

$$
\vec{p} \times \vec{\gamma} \in(L \times \mathcal{L}(L))^{h} .
$$

It is enough to show that the action of $\pi_{1}\left(\mathcal{H}_{l, \boldsymbol{k}}^{g, h}(M, L, \boldsymbol{b}), u_{0}\right)$ on the group $\mathbb{Z}_{u_{0}}$ induced by the system $\mathcal{Z}_{w_{1}\left(\operatorname{det}\left(D_{(E, F)}\right)\right)}$ is the same as the one induced by the pulled-back system $\mathrm{ev}^{*} \mathcal{Z}_{\left(w_{1}, w_{2}\right)}^{F}$.

By definition, the action induced by $\mathcal{Z}_{w_{1}\left(\operatorname{det}\left(D_{(E, F)}\right)\right)}$ is given by $\left\langle w_{1}\left(\operatorname{det}\left(D_{(E, F)}\right)\right), \gamma\right\rangle$ for $\gamma \in \pi_{1}\left(\mathcal{H}_{l, \boldsymbol{k}}^{g, h}(M, L, \boldsymbol{b}), u_{0}\right)$. By Corollary 1.5,

$$
\left\langle w_{1}\left(\operatorname{det} D_{(E, F)}\right), \gamma\right\rangle=\sum_{i=1}^{h}\left(\left\langle w_{1}(F), b_{i}\right\rangle+1\right)\left\langle w_{1}(F), \alpha_{i}\right\rangle+\sum_{i=1}^{h}\left\langle w_{2}(F), \beta_{i}\right\rangle,
$$

where $\beta_{i}$ is the torus in $L$ traced by the $i^{\text {th }}$ boundary $(\partial \Sigma)_{i}$ and $\alpha_{i}$ is the loop in $L$ traced by the boundary marked point $x_{1, i}$. 
The action of $\gamma \in \pi_{1}\left(\mathcal{H}_{l, \boldsymbol{k}}^{g, h}(M, L, \boldsymbol{b}), u_{0}\right)$ induced by the pullback system is by definition the action of the image $\widetilde{\gamma} \in \pi_{1}\left((L \times \mathcal{L}(L))^{h},\left(\vec{p}_{0}, \vec{\gamma}_{0}\right)\right)$ of $\gamma$ under the map ev. By construction, it is given by

$$
\sum_{i=1}^{h}\left(\left\langle w_{1}(F), \gamma_{i}\right\rangle+1\right)\left\langle w_{1}(F), \alpha_{i}\right\rangle+\sum_{i=1}^{h}\left\langle w_{2}(F), \beta_{i}\right\rangle .
$$

This shows the two actions are the same.

Lemma 4.7 Let f: $\mathcal{H}_{l, \mathbf{1}}^{g, h}(M, L, \boldsymbol{b}) \rightarrow \mathcal{H}_{l, \mathbf{0}}^{g, h}(M, L, \boldsymbol{b})$ be the map forgetting the boundary marked points. The system $\mathrm{ev}^{*} \mathcal{Z}_{\left(w_{1}, w_{2}\right)}^{F}$ pushes down to a system

$$
\widetilde{\mathrm{ev}}^{*} \mathcal{Z}_{\left(w_{1}, w_{2}\right)}^{F}=\mathfrak{f}_{*} \circ \mathrm{ev}^{*} \mathcal{Z}_{\left(w_{1}, w_{2}\right)}^{F}
$$

over $\mathcal{H}_{l, \mathbf{0}}^{g, h}(M, L, \boldsymbol{b})$ isomorphic to $\mathcal{Z}_{w_{1}\left(\operatorname{det} D_{(E, F)}\right)}$.

Proof It is enough to show that the system pushes down under the map forgetting the boundary points on a particular boundary component $(\partial \Sigma)_{i}$. Since the fiber of the forgetful map is connected, we need to show only that the system is trivial along the fiber. The fiber is homotopic to $S^{1}$. Let $\gamma$ be a loop in the fiber. Its image under the map ev is a degenerate torus, since the image of any point in the fiber is the same loop in $L$ up to reparameterization. Thus, the $w_{2}(F)$ term in (4-1) vanishes. The image that a point on the boundary traces along the loop is the boundary itself, and therefore the remaining term in (4-1) becomes

$$
\left(\left\langle w_{1}(F), b_{i}\right\rangle+1\right)\left\langle w_{1}(F), b_{i}\right\rangle \equiv 0 .
$$

Thus, the system is trivial along the fiber. Since $\pi_{1}\left(\mathcal{H}_{l, \mathbf{1}}^{g, h}(M, L, \boldsymbol{b})\right)$ surjects on $\pi_{1}\left(\mathcal{H}_{l, \mathbf{0}}^{g, h}(M, L, \boldsymbol{b})\right)$, every loop $\gamma$ in $\mathcal{H}_{l, \mathbf{0}}^{g, h}(M, L, \boldsymbol{b})$ lifts to a loop $\widetilde{\gamma}$ in $\mathcal{H}_{l, \mathbf{1}}^{g, h}(M, L, \boldsymbol{b})$ and

$$
\left\langle w_{1}\left(\operatorname{det}\left(D_{(E, F)}\right)\right), \gamma\right\rangle=\left\langle w_{1}\left(\operatorname{det}\left(D_{(E, F)}\right)\right), \widetilde{\gamma}\right\rangle .
$$

By Proposition 4.6, the induced action of $\mathcal{Z}_{w_{1}\left(\operatorname{det}\left(D_{(E, F)}\right)\right)}$ on $\pi_{1}\left(\mathcal{H}_{l, \mathbf{1}}^{g, h}(M, L, \boldsymbol{b})\right)$ is the same as the one induced by $\operatorname{ev}^{*} \mathcal{Z}_{\left(w_{1}, w_{2}\right)}^{F}$. Thus, the local systems $\widetilde{\mathrm{ev}}^{*} \mathcal{Z}_{\left(w_{1}, w_{2}\right)}^{F}$ and $\mathcal{Z}_{w_{1}\left(\operatorname{det} D_{(E, F)}\right)}$ are isomorphic.

Remark 4.8 An isomorphism between

$$
\operatorname{ev}^{*} \mathcal{Z}_{\left(w_{1}, w_{2}\right)}^{F} \quad \text { and } \quad \mathcal{Z}_{w_{1}\left(\operatorname{det} D_{(E, F)}\right)}
$$

over $\mathcal{H}_{l, \mathbf{1}}^{g, h}(M, L, \boldsymbol{b})$ determines an isomorphism between their pushdowns by $\mathfrak{f}$ over $\mathcal{H}_{l, \mathbf{0}}^{g, h}(M, L, \boldsymbol{b})$ since the systems are trivial over every fiber of $\mathfrak{f}$. 
In order to describe an isomorphism between the local systems of Proposition 4.6, we choose a trivialization of the determinant line $\operatorname{det}\left(D_{(E, F)}\right)$ over the element $u_{0}$ of $\mathcal{H}_{l, \boldsymbol{k}}^{g, h}(M, L, \boldsymbol{b})$ used in the proof of Proposition 4.6. This fixes the group $\mathbb{Z}$ at $u_{0}$ and thus an isomorphism between the two systems.

Proposition 4.9 A trivialization of $\operatorname{det}\left(D_{(E, F)}\right)_{\mid u_{0}}$ is induced by trivializations of $F^{1}=\operatorname{det}(F)$ over $u_{0}\left(x_{1, i}\right)$ for some $x_{1, i} \in(\partial \Sigma)_{i}$ and trivializations of $\widetilde{F}=F \oplus 3 F^{1}$ over $u_{0}\left((\partial \Sigma)_{i}\right)$ for $i=1, \ldots, h$. The effect on the orientation of $\operatorname{det}\left(D_{(E, F)}\right)_{\mid u_{0}}$ under the changes $s_{i} \widetilde{F} \in \pi_{1}(\mathrm{SO}(n)) \cong\{0,1\}$ and $o_{i} F^{1}$ in the trivializations of $\widetilde{F}_{\mid u_{0}\left((\partial \Sigma)_{i}\right)}$ and of $F_{\mid u_{0}\left(x_{1, i}\right)}$ is the multiplication by $(-1)^{\epsilon}$, where

$$
\epsilon=s_{i}^{\widetilde{F}}+\left(\left\langle w_{1}\left(F^{1}\right), b_{i}\right\rangle+1\right) o_{i}^{F^{1}} .
$$

Proof By (2-1), we have a canonical isomorphism

$$
\operatorname{det}\left(D_{(E, F)}\right) \otimes \operatorname{det}\left(\bar{\partial}_{\left(4 E^{1}, 4 F^{1}\right)}\right) \cong \operatorname{det}\left(\bar{\partial}_{(\widetilde{E}, \widetilde{F})}\right) \otimes \operatorname{det}\left(\bar{\partial}_{\left(E^{1}, F^{1}\right)}\right) .
$$

Thus, a choice of trivializations over $u_{0}$ each of of $\operatorname{det}\left(\bar{\partial}_{\left(4 E^{1}, 4 F^{1}\right)}\right), \operatorname{det}(\bar{\partial}(\widetilde{E}, \widetilde{F}))$ and $\operatorname{det}\left(\bar{\partial}_{\left(E^{1}, F^{1}\right)}\right)$ induces one on $\operatorname{det}\left(D_{(E, F)}\right)$.

By the proof of Proposition $3.1, \operatorname{det}(\bar{\partial}(\widetilde{E}, \widetilde{F}))_{\mid u_{0}}$ is canonically isomorphic to the determinant lines of operators over disks tensored with the determinant line over a closed surface and the top exterior powers of complex bundles. The last two have canonical orientations coming from the complex structures, and thus we only need to choose a trivialization of the determinants over the disks. The bundle pairs over the disks are trivial. A trivialization of $\widetilde{F}$ over each $(\partial \Sigma)_{i}$ determines a trivialization of $(\widetilde{E}, \widetilde{F})$ over the corresponding disk $D_{i}^{2}$, uniquely up to homotopy; see the proof of Lemma 3.4. The resulting trivialization of $(\widetilde{E}, \widetilde{F})$ identifies each determinant line with the determinant line of the standard Cauchy-Riemann operator over the disk, which is canonically oriented. This implies that a choice of trivializations of $\widetilde{F}$ over $u_{0}\left((\partial \Sigma)_{i}\right)$, with $i=1, \ldots, h$, induces a trivialization of the determinant line $\operatorname{det}(\bar{\partial}(\widetilde{E}, \widetilde{F}))_{\mid u_{0}}$. Changing the homotopy type of the trivialization of $\widetilde{F}_{\mid u_{0}\left((\partial \Sigma)_{i}\right)}$ changes the induced orientation of the determinant line over $D_{i}^{2}$ and thus of $\operatorname{det} \bar{\partial}_{(\widetilde{E}, \widetilde{F})}$; see the proof of Lemma 3.4.

Likewise, a choice of trivializations of $4 F^{1}$ over $u_{0}\left((\partial \Sigma)_{i}\right)$, with $i=1, \ldots, h$, induces a trivialization of the determinant line $\operatorname{det}\left(\bar{\partial}_{\left(4 E^{1}, 4 F^{1}\right)}\right)$. However, the bundle $4 F^{1}$ has a canonical (up to homotopy) trivialization over each $(\partial \Sigma)_{i}$, which is induced by any trivialization of $2 F^{1}$ over $(\partial \Sigma)_{i}$. Thus, $\operatorname{det} \bar{\partial}_{\left(4 E^{1}, 4 F^{1}\right)}$ has a canonical orientation.

By the proof of Proposition 3.2, $\operatorname{det}\left(\bar{\partial}_{\left(E^{1}, F^{1}\right)}\right)_{\mid u_{0}}$ is isomorphic to the determinant lines of operators over disks tensored with the determinant line over a closed surface and the top exterior powers of complex vector bundles. The last two are canonically 
oriented, and again we only need to choose a trivialization of the determinant lines over the disks. By Lemma 3.5, if $\left\langle w_{1}\left(F^{1}\right), b_{i}\right\rangle=0$, the index of the operator on $D_{i}^{2}$ is isomorphic to $F_{\mid u_{0}\left(x_{1, i}\right)}^{1}$. Hence, a choice of a trivialization of $F_{\mid u_{0}\left(x_{1, i}\right)}^{1}$ induces a trivialization of its determinant line; changing the homotopy type of the trivialization of $F_{\mid u_{0}\left(x_{1, i}\right)}^{1}$ changes the induced orientation of the determinant line over $D_{i}^{2}$ and thus also of $\operatorname{det} \bar{\partial}_{\left(E^{1}, F^{1}\right)}$. If $\left\langle w_{1}\left(F^{1}\right), b_{i}\right\rangle=1$, the index is isomorphic to the direct sum of the fibers of $F^{1}$ over the images of two points $x_{1, i}, x_{2, i} \in(\partial \Sigma)_{i}$. We can use the orientation of the boundary of $\Sigma$ to transport a choice of a trivialization of $F_{\mid u_{0}\left(x_{1, i}\right)}^{1}$ to $F_{\mid u_{0}\left(x_{2, i}\right)}^{1}$. In this way again, a choice of trivializations of $F_{\mid u_{0}\left(x_{1, i}\right)}^{1}$ determines trivializations of the determinant lines of the operators on the disks. However, in this case, changing the homotopy type of the trivialization of $F_{\mid u_{0}\left(x_{1, i}\right)}^{1}$ does not change the induced orientation of the determinant line over $D_{i}^{2}$ and thus of $\operatorname{det} \bar{\partial}_{\left(E^{1}, F^{1}\right)}$.

Proof of Theorem 1.7 Suppose there is at least one boundary marked point on each boundary component of $\Sigma$. By Proposition 4.6, there is an isomorphism

$$
\mathfrak{p}: \mathcal{Z}_{w_{1}\left(\operatorname{det} D_{(E, F)}\right)} \cong \mathrm{ev}^{*} \mathcal{Z}_{\left(w_{1}, w_{2}\right)}^{F} .
$$

By Proposition 4.9, a choice of a basepoint $u_{0} \in \mathcal{H}_{l, \boldsymbol{k}}^{g, h}(M, L, \boldsymbol{b})$, with image under the map ev equal to the basepoint of $(L \times \mathcal{L}(L))^{h}$, determines an isomorphism. We claim that the isomorphism is independent of the choice of such $u_{0}$. Let us first describe the isomorphism $\mathfrak{p}$ for a given $u_{0}$. The trivializations of $\widetilde{F}$ and $F^{1}$ over $u_{0}\left((\partial \Sigma)_{i}\right)$ and $u_{0}\left(x_{1, i}\right)$, for $i=1, \ldots, h$, respectively, induce an isomorphism $\mathcal{Z}_{w_{1}\left(\operatorname{det} D_{(E, F)}\right) \mid u_{0}} \cong \mathbb{Z}$. By construction $\mathrm{ev}^{*} \mathcal{Z}_{\left(w_{1}, w_{2}\right) \mid u_{0}}^{F}=\mathbb{Z}$ and

$$
\mathcal{Z}_{w_{1}\left(\operatorname{det} D_{(E, F)}\right) \mid u_{0}} \cong \mathbb{Z} \stackrel{\mathfrak{p}}{\longrightarrow} \mathbb{Z}=\operatorname{ev}^{*} \mathcal{Z}_{\left(w_{1}, w_{2}\right) \mid u_{0}}^{F}, \quad \mathfrak{p}(1)=1 .
$$

If $v \in \mathcal{Z}_{w_{1}\left(\operatorname{det} D_{(E, F)}\right) \mid u}$, then $\mathfrak{p}(v)=\psi_{\gamma^{-1}} \mathfrak{p}\left(\phi_{\gamma} v\right)$, where $\gamma$ is a path from $u$ to $u_{0}$, $\phi_{\gamma}$ is the isomorphism induced by the path in the system $\mathcal{Z}_{w_{1}\left(\operatorname{det} D_{(E, F)}\right)}$, and $\psi_{\gamma}$ is the isomorphism in $\mathrm{ev}^{*} \mathcal{Z}_{\left(w_{1}, w_{2}\right)}$. This is independent of the path $\gamma$.

Let $u_{0}^{\prime}$ be another point, with image under the map ev equal to the basepoint of $(L \times \mathcal{L}(L))^{h}$, and denote by $\mathfrak{p}^{\prime}$ the induced isomorphism. We show that

$$
\mathfrak{p}(v)=\mathfrak{p}^{\prime}(v) \quad \forall u \in \mathcal{H}_{l, \boldsymbol{k}}^{g, h}(M, L, \boldsymbol{b}), v \in \mathcal{Z}_{w_{1}\left(\operatorname{det} D_{(E, F)}\right) \mid u} .
$$

It is enough to confirm this equality for $u=u_{0}^{\prime}$ and $v=1 \in \mathcal{Z}_{w_{1}\left(\operatorname{det} D_{(E, F)}\right) \mid u_{0}^{\prime}} \cong \mathbb{Z}$. Since ev $\left(u_{0}\right)=\operatorname{ev}\left(u_{0}^{\prime}\right)$, the isomorphism

$$
\mathbb{Z}=\mathcal{Z}_{w_{1}\left(\operatorname{det} D_{(E, F)}\right) \mid u_{0}^{\prime}} \cong \mathcal{Z}_{w_{1}\left(\operatorname{det} D_{(E, F)}\right) \mid u_{0}}=\mathbb{Z},
$$


induced by a path $\gamma$ between $u_{0}^{\prime}$ and $u_{0}$ is given by multiplication with $(-1)^{\epsilon}$, where

$$
\epsilon=\sum_{i=1}^{h}\left(\left\langle w_{1}(F), b_{i}\right\rangle+1\right)\left\langle w_{1}(F), \operatorname{ev}_{x_{1, i}}(\gamma)\right\rangle+\sum_{i=1}^{h}\left\langle w_{2}(F), \operatorname{ev}_{i}(\gamma)\right\rangle,
$$

since this expression traces the change in trivializations of $\widetilde{F}$ and $F^{1}$. By definition, the isomorphism $\psi_{\gamma}$ equals $\varphi_{\operatorname{ev}(\gamma)}$, where $\varphi_{\operatorname{ev}(\gamma)}$ is the isomorphism in $\mathcal{Z}_{\left(w_{1}, w_{2}\right)}^{F}$ corresponding to the loop $\operatorname{ev}(\gamma)$. By definition, $\varphi_{\mathrm{ev}}(\gamma)$ is also the multiplication by $(-1)^{\epsilon}$. Therefore,

$$
\mathfrak{p}(v)=\psi_{\gamma^{-1}} \mathfrak{p}\left(\phi_{\gamma}(1)\right)=\varphi_{\mathrm{ev}(\gamma)} \mathfrak{p}\left((-1)^{\epsilon} \cdot 1\right)=(-1)^{\epsilon}(-1)^{\epsilon} \mathfrak{p}(1)=1=\mathfrak{p}^{\prime}(v) .
$$

Lemma 4.7 and Remark 4.8 now conclude the proof of Theorem 1.7.

Remark 4.10 We can choose the basepoint of $\mathcal{H}_{l, \boldsymbol{k}}^{g, h}(M, L, \boldsymbol{b})$ for different $(l, \boldsymbol{k})$ in a systematic way, thus fixing an isomorphism

$$
\mathfrak{f}^{*} \mathcal{Z}_{w_{1}\left(\operatorname{det}\left(D_{(E, F)}\right)\right)} \cong \mathcal{Z}_{w_{1}\left(\operatorname{det}\left(D_{(E, F)}\right)\right)} .
$$

We first choose elements in $\mathfrak{B}_{l, \boldsymbol{k}}^{g, h}(M, L, \boldsymbol{b}) \times \mathcal{J}_{\Sigma}$ inductively. Let $u_{0} \in \mathfrak{B}_{0, \boldsymbol{0}}^{g, h}(\Sigma, \boldsymbol{b}) \times \mathcal{J}_{\Sigma}$ be a map with $u_{0 \mid(\partial \Sigma)_{i}}=\gamma_{i}$. If we have chosen an element in $\mathfrak{B}_{l, \boldsymbol{k}}^{g, h}(M, L, \boldsymbol{b}) \times \mathcal{J}_{\Sigma}$, select an element in the space with an additional marked point $\mathfrak{B}_{l^{\prime}, \boldsymbol{k}}^{g, h}(\Sigma, \boldsymbol{b}) \times \mathcal{J}_{\Sigma}$, $l^{\prime}+\left|\boldsymbol{k}^{\prime}\right|=l+|\boldsymbol{k}|+1$, by adding a marked point to the given collection. We choose the basepoint $\left[u_{0}, \boldsymbol{z}, \boldsymbol{x}_{\mathbf{1}}, \ldots, \boldsymbol{x}_{\boldsymbol{h}}\right]$ of $\mathcal{H}_{l, \boldsymbol{k}}^{g, h}(M, L, \boldsymbol{b})$ to be the class of the chosen element in $\mathfrak{B}_{l, \boldsymbol{k}}^{g, h}(M, L, \boldsymbol{b}) \times \mathcal{J}_{\Sigma}$ and construct the map

$$
\text { ev: } \mathcal{H}_{l, \boldsymbol{k}}^{g, h}(M, L, \boldsymbol{b}) \rightarrow(L \times \mathcal{L}(L))^{h}
$$

to send $\left[u_{0}, \boldsymbol{z}, \boldsymbol{x}_{\mathbf{1}}, \ldots, \boldsymbol{x}_{\boldsymbol{h}}\right]$ to the basepoint of the corresponding component.

Proof of Corollary 1.8 Suppose the moduli space of maps $\mathfrak{M}_{l, \boldsymbol{k}}^{g, h}(M, L, \boldsymbol{b})$ and the moduli space of domains $\mathfrak{M}_{l, \boldsymbol{k}}^{g, h}$ are manifolds. The map

$$
f: \mathfrak{M}_{l, \boldsymbol{k}}^{g, h}(M, L, \boldsymbol{b}) \rightarrow \mathfrak{M}_{l, \boldsymbol{k}}^{g, h}
$$

then induces a canonical isomorphism

$$
\begin{aligned}
\bigwedge^{\text {top }} T \mathfrak{M}_{l, \boldsymbol{k}}^{g, h}(M, L, \boldsymbol{b}) & \cong f^{*} \bigwedge^{\text {top }} T \mathfrak{M}_{l, \boldsymbol{k}}^{g, h} \otimes \bigwedge^{\text {top }} T \mathfrak{M}_{l, \boldsymbol{k}}^{g, h}(M, L, \boldsymbol{b})^{\text {Vert }} \\
& \cong f^{*} \bigwedge^{\text {top }} T \mathfrak{M}_{l, \boldsymbol{k}}^{g, h} \otimes \bigwedge^{\text {top }} \operatorname{ker} D_{\bar{\partial}} .
\end{aligned}
$$

Thus, the local system of orientations on the moduli space of maps is the restriction of the local system of orientations of the bundle

$$
f^{*} \bigwedge^{\text {top }} \mathfrak{M}_{l, \boldsymbol{k}}^{g, h} \otimes \operatorname{det} D_{\bar{\partial}} \rightarrow \mathcal{H}_{l, \boldsymbol{k}}^{g, h}(M, L, \boldsymbol{b}) .
$$


The moduli space of domains, $\mathfrak{M}_{l, \boldsymbol{k}}^{g, h}$, is canonically oriented as follows. Let $\mathfrak{M}_{l, \boldsymbol{k}^{\prime}}^{g, h}$ be the moduli space with one more boundary marked point. The map $\mathfrak{f}$ forgetting the additional marked point induces a canonical isomorphism

$$
\bigwedge^{\text {top }} T \mathfrak{M}_{l, \boldsymbol{k}^{\prime}}^{g, h} \cong \mathfrak{f}^{*} \bigwedge^{\mathrm{top}} T \mathfrak{M}_{l, \boldsymbol{k}}^{g, h} \otimes \bigwedge^{\mathrm{top}}\left(T \mathfrak{M}_{l, \boldsymbol{k}^{\prime}}^{g, h}\right)^{\text {Vert }}
$$

The fiber of the map forgetting the boundary marked point is canonically isomorphic to a subset of the boundary component the point lies on and is thus canonically oriented. The transition maps of $\bigwedge^{\text {top }}\left(T \mathfrak{M}_{l, \boldsymbol{k}^{\prime}}^{g, h}\right)^{\text {Vert }}$ are diffeomorphisms of the domain preserving the orientation and each boundary component. Therefore, the bundle $\bigwedge^{\text {top }}\left(T \mathfrak{M}_{l, \boldsymbol{k}^{\prime}}^{g, h}\right)^{\text {Vert }}$ is orientable and canonically oriented by the orientation of a fiber. This implies that if either $\mathfrak{M}_{l, \boldsymbol{k}}^{g, h}$ or $\mathfrak{M}_{l, \boldsymbol{k}^{\prime}}^{g, h}$ has a canonical orientation, so does the other. By Ivashkovich and Shevchishin [6], the moduli space $\mathfrak{M}_{l, \boldsymbol{k}}^{g, h}$, with $\boldsymbol{k}=(1, \ldots, 1)$, possesses a holomorphic structure and in particular is canonically oriented. Therefore, $\mathfrak{M}_{l, \boldsymbol{k}}^{g, h}$ is canonically oriented and the orientation is compatible with the maps forgetting the marked points. Thus, the bundle $f^{*} \wedge^{\text {top }} \mathfrak{M}_{l, \boldsymbol{k}}^{g, h} \otimes \operatorname{det} D_{\bar{\partial}}$ is canonically isomorphic to det $D_{\bar{\partial}}$. Theorem 1.7 now implies the result.

The case when $\mathfrak{M}_{l, \boldsymbol{k}}^{g, h}(M, L, \boldsymbol{b})$ is a manifold but $\mathfrak{M}_{l, \boldsymbol{k}}^{g, h}$ is not can be treated as follows. Consider the moduli space $\mathfrak{M}_{l^{\prime}, \boldsymbol{k}^{\prime}}^{g, h}(M, L, \boldsymbol{b})$ with enough marked points so that $\mathfrak{M}_{l^{\prime}, \boldsymbol{k}^{\prime}}^{g, h}$ is a manifold. Then,

$$
\bigwedge^{\text {top }} T \mathfrak{M}_{l^{\prime}, \boldsymbol{k}^{\prime}}^{g, h}(M, L, \boldsymbol{b}) \cong \operatorname{det} D_{\bar{\partial}} .
$$

Moreover, the map forgetting the additional marked points

$$
\mathfrak{f}: \mathfrak{M}_{l^{\prime}, \boldsymbol{k}^{\prime}}^{g, h}(M, L, \boldsymbol{b}) \rightarrow \mathfrak{M}_{l, \boldsymbol{k}}^{g, h}(M, L, \boldsymbol{b})
$$

induces a canonical isomorphism

$$
\bigwedge^{\text {top }} T \mathfrak{M}_{l^{\prime}, \boldsymbol{k}^{\prime}}^{g, h}(M, L, \boldsymbol{b}) \cong \mathfrak{f}^{*} \bigwedge^{\text {top }} T \mathfrak{M}_{l, \boldsymbol{k}}^{g, h}(M, L, \boldsymbol{b}) \otimes \bigwedge^{\text {top }}\left(T \mathfrak{M}_{l^{\prime}, \boldsymbol{k}^{\prime}}^{g, h}(M, L, \boldsymbol{b})\right)^{\text {Vert }} \text {. }
$$

As above, the bundle $\bigwedge^{\text {top }}\left(T \mathfrak{M}_{l^{\prime}, \boldsymbol{k}^{\prime}}^{g, h}(M, L, \boldsymbol{b})\right)^{\text {Vert }}$ is canonically oriented and thus,

$$
\mathfrak{f}^{*} \bigwedge^{\text {top }} T \mathfrak{M}_{l, \boldsymbol{k}}^{g, h}(M, L, \boldsymbol{b}) \cong \bigwedge^{\text {top }} T \mathfrak{M}_{l^{\prime}, \boldsymbol{k}^{\prime}}^{g, h}(M, L, \boldsymbol{b}) \cong \operatorname{det} D_{\bar{\partial}} \cong \mathfrak{f}^{*} \operatorname{det} D_{\bar{\partial}} .
$$

Since $\mathfrak{f}$ is surjective on $\pi_{1}$,

$$
\bigwedge^{\text {top }} T \mathfrak{M}_{l, \boldsymbol{k}}^{g, h}(M, L, \boldsymbol{b}) \cong \operatorname{det} D_{\bar{\partial}} ;
$$

see the proof of Lemma 4.7. The result follows from Theorem 1.7.

Proof of Corollary 1.10 The line bundles

$$
\bigwedge_{\mathbb{R}}^{\text {top }} \mathcal{V}_{n ; \boldsymbol{a}}^{\mathbb{R}} \cong \operatorname{det} \bar{\partial}_{\left(\mathcal{L}_{n, \boldsymbol{a}}, \mathcal{L}_{n, \boldsymbol{a}}^{\mathbb{R}}\right)}, \quad \bigwedge_{\mathbb{R}}^{\mathrm{top}} T \mathfrak{M}_{l, \boldsymbol{k}}^{g, h}\left(\mathbb{C} \mathbb{P}^{n}, \mathbb{R P}^{n}, \boldsymbol{b}\right) \rightarrow \mathfrak{M}_{l, \boldsymbol{k}}^{g, h}\left(\mathbb{C} \mathbb{P}^{n}, \mathbb{R} \mathbb{P}^{n}, \boldsymbol{b}\right)
$$


are isomorphic if their first Stiefel-Whitney classes evaluate to the same number over every loop $\gamma$. By Corollary 1.5, the first Stiefel-Whitney class of $\mathcal{V}_{n, \boldsymbol{a}}$ evaluated on a loop $\gamma$ is given by

$$
\sum_{i=1}^{h}\left(\left\langle w_{1}\left(\mathcal{L}_{n, \boldsymbol{a}}^{\mathbb{R}}\right), b_{i}\right\rangle+1\right)\left\langle w_{1}\left(\mathcal{L}_{n, \boldsymbol{a}}^{\mathbb{R}}\right), \operatorname{ev}_{x_{1, i}}(\gamma)\right\rangle+\sum_{i=1}^{h}\left\langle w_{2}\left(\mathcal{L}_{n, \boldsymbol{a}}^{\mathbb{R}}\right), \operatorname{ev}_{i}(\gamma)\right\rangle .
$$

Let $\mathcal{O}^{\mathbb{R}}(a)$ denote the tensor product of $a$ copies of the tautological line bundle over $\mathbb{R} \mathbb{P}^{n}$ and $\eta=w_{1}\left(\mathcal{O}^{\mathbb{R}}(1)\right)$ be the generator of $H^{1}\left(\mathbb{R P}^{n}, \mathbb{Z}_{2}\right)$. Since

$$
\mathcal{L}_{n, \boldsymbol{a}}^{\mathbb{R}}=\bigoplus_{i} \mathcal{O}^{\mathbb{R}}\left(a_{i}\right)
$$

we have

$$
w_{1}\left(\mathcal{L}_{n, \boldsymbol{a}}^{\mathbb{R}}\right)=\sum_{i=1}^{m} a_{i} \eta, \quad w_{2}\left(\mathcal{L}_{n, \boldsymbol{a}}^{\mathbb{R}}\right)=\sum_{i, j}^{m} a_{i} a_{j} \eta^{2}
$$

Since $w_{2}\left(\mathcal{L}_{n, \boldsymbol{a}}^{\mathbb{R}}\right)$ is a square of a class, it evaluates to zero on each torus $\operatorname{ev}_{i}(\gamma)$. By Corollary 1.5 , the first Stiefel-Whitney class of $\mathfrak{M}_{l, \boldsymbol{k}}^{g, h}\left(\mathbb{C} \mathbb{P}^{n}, \mathbb{R P}^{n}, \boldsymbol{b}\right)$ evaluated on the loop $\gamma$ is given by (4-2) with $\mathcal{L}_{n, \boldsymbol{a}}^{\mathbb{R}}$ replaced by $T \mathbb{R} \mathbb{P}^{n}$. Since $w_{2}\left(\mathbb{R} \mathbb{P}^{n}\right)$ is a square of a class, the second sum vanishes in this case as well. The condition $n-\sum a_{i}$ is odd implies that

$$
w_{1}\left(\mathcal{L}_{n, \boldsymbol{a}}^{\mathbb{R}}\right)=(n+1) \eta=w_{1}\left(\mathbb{R} \mathbb{P}^{n}\right)
$$

Thus, the two line bundles are isomorphic.

By Proposition 4.9, a choice of trivializations of $\mathcal{L}_{n, \boldsymbol{a}}^{\mathbb{R}} \oplus 3 \operatorname{det}\left(\mathcal{L}_{n, \boldsymbol{a}}^{\mathbb{R}}\right)$ and $\operatorname{det}\left(\mathcal{L}_{n, \boldsymbol{a}}^{\mathbb{R}}\right)$ over $\operatorname{ev}_{i}\left(u_{0}\right)$ and $u_{0}\left(x_{1, i}\right)$, respectively, with $i=1, \ldots, h$, determines a trivialization $\operatorname{det} \bar{\partial}_{\left(\mathcal{L}_{n, \boldsymbol{a}}, \mathcal{L}_{n, \boldsymbol{a}}^{\mathbb{R}}\right) \mid u_{0}} \cong \mathbb{R}$. Similarly, a trivialization

$$
\bigwedge_{\mathbb{R}}^{\text {top }} T \mathfrak{M}_{l, \boldsymbol{k}}^{g, h}\left(\mathbb{C} \mathbb{P}^{n}, \mathbb{R} \mathbb{P}^{n}, \boldsymbol{b}\right)_{\mid u_{0}} \cong \mathbb{R}
$$

is determined by a choice of trivializations of $T \mathbb{R} \mathbb{P}^{n} \oplus 3 \operatorname{det}\left(T \mathbb{R} \mathbb{P}^{n}\right)$ and $\operatorname{det}\left(T \mathbb{R P}^{n}\right)$ over $\operatorname{ev}_{i}\left(u_{0}\right)$ and $u_{0}\left(x_{1, i}\right)$, respectively, with $i=1, \ldots, h$.

If $\mathcal{O}^{\mathbb{R}}$ denotes the trivial line bundle, there are canonical isomorphisms

$$
\mathcal{O}^{\mathbb{R}} \oplus T \mathbb{R} \mathbb{P}^{n} \cong(n+1) \mathcal{O}^{\mathbb{R}}(1), \quad \operatorname{det}\left(T \mathbb{R} \mathbb{P}^{n}\right) \cong \mathcal{O}^{\mathbb{R}}(n+1)
$$

Thus, a choice of a trivialization of $(n+1) \mathcal{O}^{\mathbb{R}}(1) \oplus 3 \mathcal{O}^{\mathbb{R}}(n+1)$ over $\operatorname{ev}_{i}\left(u_{0}\right)$ determines one on $T \mathbb{R} \mathbb{P}^{n} \oplus 3 \operatorname{det}\left(T \mathbb{R} \mathbb{P}^{n}\right)$. A choice of a trivialization of $\mathcal{O}^{\mathbb{R}}(1)$ over $u_{0}\left(x_{1, i}\right)$ and the canonical trivialization

$$
2 \mathcal{O}^{\mathbb{R}}(1) \cong \mathcal{O}^{\mathbb{R}} \oplus T \mathbb{R} \mathbb{P}^{1} \cong 2 \mathcal{O}^{\mathbb{R}}
$$


over $\operatorname{ev}_{i}\left(u_{0}\right)$ determine an isomorphism

$$
\begin{aligned}
(n+1) \mathcal{O}^{\mathbb{R}}(1) \oplus 3 \mathcal{O}^{\mathbb{R}}(n+1) & \\
& \cong \begin{cases}\frac{n}{2}\left(2 \mathcal{O}^{\mathbb{R}}\right) \oplus \mathcal{O}^{\mathbb{R}}(1) \oplus 3 \mathcal{O}^{\mathbb{R}}(1) \cong(n+4) \mathcal{O}^{\mathbb{R}} & \text { if } 2 \mid n, \\
\frac{n+1}{2}\left(2 \mathcal{O}^{\mathbb{R}}\right) \oplus 3 \mathcal{O}^{\mathbb{R}} & \text { if } 2 \nmid n,\end{cases}
\end{aligned}
$$

over $\mathrm{ev}_{i}\left(u_{0}\right)$. A nontrivial change in the trivialization of $\mathcal{O}^{\mathbb{R}}(1)$ over $u_{0}\left(x_{1, i}\right)$ does not affect the trivialization of $3 \mathcal{O}^{\mathbb{R}}(n+1)$ in (4-3) when $n$ is odd. Thus, the trivialization in (4-3) is canonical. A choice of a trivialization of $\mathcal{O}^{\mathbb{R}}(1)$ over $u_{0}\left(x_{1, i}\right)$ determines one on $\mathcal{O}^{\mathbb{R}}(n+1) \cong \operatorname{det}\left(T \mathbb{R} \mathbb{P}^{n}\right)$. A nontrivial change in the trivialization of $\mathcal{O}^{\mathbb{R}}(1)$ over $u_{0}\left(x_{1, i}\right)$ results in a nontrivial change of trivialization of $\mathcal{O}^{\mathbb{R}}(n+1)$ over $u_{0}\left(x_{1, i}\right)$ if and only if $n$ is even.

There are canonical isomorphisms

$$
\mathcal{L}_{n, \boldsymbol{a}}^{\mathbb{R}} \cong(m-\widetilde{m}) \mathcal{O}^{\mathbb{R}} \oplus \widetilde{m} \mathcal{O}^{\mathbb{R}}(1), \quad \operatorname{det}\left(\mathcal{L}_{n, \boldsymbol{a}}^{\mathbb{R}}\right) \cong \mathcal{O}^{\mathbb{R}}(\widetilde{m}),
$$

where $\widetilde{m}$ is the number of odd $a_{i}$. By (4-3), $\mathcal{L}_{n, \boldsymbol{a}}^{\mathbb{R}} \oplus 3 \operatorname{det}\left(\mathcal{L}_{n, \boldsymbol{a}}^{\mathbb{R}}\right)$ has a canonical trivialization over $\operatorname{ev}_{i}\left(u_{0}\right)$. A trivialization of $\mathcal{O}^{\mathbb{R}}(1)$ over $u_{0}\left(x_{1, i}\right)$ induces one on $\mathcal{O}^{\mathbb{R}}(\widetilde{m})$, and a nontrivial change in the former results in a nontrivial change in the latter if and only if $\widetilde{m}$ is odd. Thus, a choice of trivializations of $\mathcal{O}^{\mathbb{R}}(1)$ over $u_{0}\left(x_{1, i}\right)$ determine isomorphisms

$$
\bigwedge_{\mathbb{R}}^{\text {top }} T \mathfrak{M}_{l, \boldsymbol{k}}^{g, h}\left(\mathbb{C} \mathbb{P}^{n}, \mathbb{R} \mathbb{P}^{n}, \boldsymbol{b}\right)_{\mid u_{0}} \cong \mathbb{R}, \quad \bigwedge_{\mathbb{R}}^{\text {top }} \mathcal{V}_{n ; \boldsymbol{a} \mid u_{0}}^{\mathbb{R}} \cong \mathbb{R}
$$

If $\widetilde{m} \equiv n+1 \bmod 2$, a change in the trivialization $\mathcal{O}^{\mathbb{R}}(1)_{\mid u_{0}\left(x_{1, i}\right)}$ affects both isomorphisms in the same way. Thus, in this case, the composite isomorphism

$$
\bigwedge_{\mathbb{R}}^{\text {top }} T \mathfrak{M}_{l, \boldsymbol{k}}^{g, h}\left(\mathbb{C} \mathbb{P}^{n}, \mathbb{R} \mathbb{P}^{n}, \boldsymbol{b}\right)_{\mid u_{0}} \cong \mathbb{R} \cong \bigwedge_{\mathbb{R}}^{\text {top }} \mathcal{V}_{n ; \boldsymbol{a} \mid u_{0}}^{\mathbb{R}}
$$

is canonical.

\section{References}

[1] G Bini, C de Concini, M Polito, C Procesi, On the work of Givental relative to mirror symmetry, Scuola Normale Superiore, Pisa (1998) MR1656956

[2] T Ekholm, J Etnyre, M Sullivan, Orientations in Legendrian contact homology and exact Lagrangian immersions, Internat. J. Math. 16 (2005) 453-532 MR2141318

[3] B Farb, D Margalit, A primer on mapping class groups, Princeton Mathematical Series 49, Princeton Univ. Press (2011) MR2850125

[4] K Fukaya, Y-G Oh, H Ohta, K Ono, Lagrangian intersection Floer theory: Anomaly and obstruction, AMS/IP Studies Adv. Math. 46, Amer. Math. Soc. (2009) 
[5] M Gromov, Pseudoholomorphic curves in symplectic manifolds, Invent. Math. 82 (1985) 307-347 MR809718

[6] S Ivashkovich, V Shevchishin, Holomorphic structure on the space of Riemann surfaces with marked boundary, Tr. Mat. Inst. Steklova 235 (2001) 98-109 MR1886576

[7] J Li, A Zinger, On the genus-one Gromov-Witten invariants of complete intersections, J. Differential Geom. 82 (2009) 641-690 MR2534991

[8] E Looijenga, Smooth Deligne-Mumford compactifications by means of Prym level structures, J. Algebraic Geom. 3 (1994) 283-293 MR1257324

[9] G Massuyeau, A short introduction to mapping class groups (2009) Available at http://www-irma.u-strasbg.fr/ massuyea/talks/MCG.pdf

[10] D McDuff, D Salamon, J-holomorphic curves and symplectic topology, Amer. Math. Soc. Colloq. Publ. 52, Amer. Math. Soc. (2004) MR2045629

[11] R Pandharipande, J Solomon, J Walcher, Disk enumeration on the quintic 3-fold, J. Amer. Math. Soc. 21 (2008) 1169-1209 MR2425184

[12] A Popa, A Zinger, Mirror symmetry for closed, open, and unoriented Gromov-Witten invariants arXiv:1010.1946

[13] P Seidel, Fukaya categories and Picard-Lefschetz theory, Europ. Math. Soc., Zürich (2008) MR2441780

[14] V de Silva, Products in the symplectic Floer homology of Lagrangian intersections, $\mathrm{PhD}$ thesis, University of Oxford (1998)

[15] J P Solomon, Intersection theory on the moduli space of holomorphic curves with Lagrangian boundary conditions, $\mathrm{PhD}$ thesis, Massachusetts Institute of Technology (2006) Available at http://search.proquest.com//docview/304947035

[16] N E Steenrod, Homology with local coefficients, Ann. of Math. 44 (1943) 610-627 MR0009114

[17] J Walcher, Evidence for tadpole cancellation in the topological string, Commun. Number Theory Phys. 3 (2009) 111-172 MR2504755

[18] K Wehrhiem, W C, Orientations for pseudoholomorphic quilts, preprint (2007) Available at http://math.mit.edu/ katrin

[19] J-Y Welschinger, Enumerative invariants of strongly semipositive real symplectic six-manifolds arXiv:0509121

Department of Mathematics, Princeton University

Princeton, NJ 08544, USA

pgeorgie@math.princeton.edu

Proposed: Yasha Eliashberg

Seconded: Gang Tian, Jim Bryan 